\title{
Reasonable adjustment in small establishments to meet accessibility requirements: A challenge for active ageing and inclusive cities. A case study of Madrid
}

\begin{abstract}
Access to and use of all activities in the built environment to ensure equal participation for people with disabilities is a basic human right, recognised globally in the Convention on the Rights of Persons with Disabilities, as well as a fundamental objective in an increasingly ageing society.
\end{abstract}

The Spanish Act on equal opportunities, non-discrimination and universal accessibility (Ley 51/2003) recognised such challenge and established the obligation to ensure accessibility in the built environment 'liable to reasonable adjustment'. The deadline for meeting that obligation was 4 December 2017.

This paper formulates a methodology to assist policy makers and urban planning authorities to define the legal concept of 'reasonable adjustment' in relation to small establishments within the existing built environment. No criteria or tools have yet been forthcoming in this area despite the deadline for compliance with the provisions of the act. That regulatory gap exacerbates the complexity in applying and interpreting the legislation on adaptations of the built environment.

Starting from a legislative and technical synopsis of the international implementation of the term 'reasonable adjustment' and based on the analysis of 120 works in establishments of Madrid and its metropolitan area, the proposed methodology addresses the characterisation of: flexibility requirements; accessibility adjustments; economic viability by activity involved; and factors affecting overall accessibility.

Regardless of the designers and owners ability, the research shows that regulatory developments, urban planning and its control by Public Authorities, both local and national, have facilitated the accessibility improvements. In terms of accessibility requirements, even today the difficulty of accessibility compliance is focused on wheelchair users. In the analysed case study, the investment in accessibility means about $0.5 \%$ of the property values.

\section{Keywords:}

Reasonable adjustment; Built environment renovation; Accessibility; Barrier-free commercial establishment; Active ageing; Inclusive cities. 


\section{Introduction}

Concern is growing around the adaptation of our environment to respond to the needs not only of people with disabilities, but also to the burgeoning community of elderly adults who often occupy living spaces scantly adapted to their circumstances. In fact, $40 \%$ of residential buildings in European Union countries were built before 1960, and almost $84 \%$ are now at least 20 years old (Directive 2010/31/EU). This means that a large majority of buildings fail to meet the basic requirements in the current legislative framework (Serrano, 2018). In Spain, the absence of such adaptations in housing and community services to ensure accessibility is one of the most frequently criticised characteristics of the built environment (Fundación ONCE, 2013 and 2015).

In 2008, the last report on disability showed that $8.5 \%$ of Spaniards living in their own homes had some kind of disability. The rate increases exponentially with age, affecting over $70 \%$ of people aged 85 or older (Observatorio Estatal de la Discapacidad, 2012). However, the trend towards ageing is far from decrease. By $2050,37 \%$ of Europeans will be over 60 . Whilst the very elderly, i.e., 80 or over, presently account for $3 \%$ of the European population, by 2050 that figure will be at least $10 \%$ in 11 European Union member states (European Union, 2002). Currently, this upward trend is ratified by the World Health Organization (2015).

In 2002, the United Nations General Assembly identified three priorities in the Political declaration and Madrid international plan of action on ageing: 'older persons and development; advancing health and well-being into old age; and ensuring that older people benefit from enabling and supportive environments'. Similarly, the European Disability Strategy (2010-2020) encourages the Union's institutions and member states to cooperate to build a barrier-free Europe for all. Such measures aim to enhance the quality of life of a growing number of European citizens and its elderly population (Jaraba, 2012).

Countless studies, conducted by accessibility promotion policies, not only focus on the paradigm of 'inclusive cities', designed for all their users, but also as a challenge in the retrofitting of obsolete neighbourhoods, which concentrate the eldest population. 'Ageing in place' (Sixsmith, 2008) is the motto for these political actions.

In 2013, Bosch highlights the need for urban planning which responds correctly to the growth of an increasingly ageing population. Older people often make heavy use of public space and services as pedestrians near their home (Berrigan, 2002), being usually the only trips they make per day outside the dwelling. In viable walks for the elderly, the public space and services must be safe and accessible, both in terms of traffic and in terms of falls or other accidents (Michael, 2006). The World Health Organization (2007) was the first to raise the concept of 'age-friendly city' for this purpose. Therefore, an age-friendly city adapts its structures and also its services to be accessible to and inclusive of older people with varying needs and capacities.

In Spain, accessibility requirements were introduced in 1982 (Ley 13/1982). In 2003, the inclusion of the concept 'reasonable adjustment' in the body of law (Ley 51/2003) affords a new opportunity to improve the existing built environment. Despite of the legal deadline (4 December 2017) laid down in a Royal Legislative Decree (Real Decreto Legislativo 1/2013), regulatory procedures have yet to be defined for many of the areas involved.

In private environments, tools have been developed to adapt housing and workplaces to people with disabilities on the grounds of economic criteria and incentives or other types of government aid (Spanish Land and Urban Regeneration Act, Royal Decree of Housing Aids, etc.). Regulatory instruments defining how the legal obligation is to be enforced to adapt areas open to the public, the object of this study, are still not in place, however.

Additionally, the difficulty inherent in intervening in existing buildings has prompted the inclusion of indeterminate flexibility provisions in the technical codes (Vega, 2012), such as 'when their application is not technically or economically viable, alternative solutions may be adopted that afford the maximum 
possible adaptation' (Real Decreto 173/2010). That fact has led to discretionary and arbitrary application of the legislation, favouring non-compliance or self-interested interpretation (Oliva, 2015).

This study is the result of a commission from the Safety and Accessibility Area, a division of the Ministry of Public Works' Directorate General for Architecture, Housing and Land, in response to a need to include the accessibility provisions of the aforementioned legal texts in the Spanish Building Code.

The results show, from the analysis of the legal concept of reasonable adjustment and for Madrid region as a methodological example: the viability of accessibility solutions, the economic capacity by activity involved to face the improvements and factors affecting overall accessibility, in order to assist policy makers and urban planning authorities to make a decision about reasonable adjustments in establishments.

\section{The term 'reasonable accommodation' or 'reasonable adjustment'}

In 2006, The Convention on the Rights of Persons with Disabilities included in the body of its text the now internationally acknowledged notion 'reasonable accommodation', defined as: 'necessary and appropriate modification and adjustments not imposing a disproportionate or undue burden, where needed in a particular case, to ensure to persons with disabilities the enjoyment or exercise on an equal basis with others of all human rights and fundamental freedoms'.

As detailed in the 2005 United Nations document, the term 'reasonable accommodation' was originally employed in United States Civil Rights Act of 1968, an anti-discrimination legislation which focus was on discrimination on the grounds of religious practice. The concept was further extended to the disability context, first in the 1973 Rehabilitation Act and subsequently in the 1990 Americans with Disabilities Act. Since then, 'reasonable accommodation' and 'undue burden' are included in US law. The 2006 Convention has incorporated these concepts, acquiring a global relevance.

The following implications can be gleaned from the studies on this legal term:

1. Needs of people with disabilities: Accommodation includes modifications, adaptations and adjustments, while also envisaging material and legislative flexibility (De Campos Velho Martel, 2011).

2. Proporcionality: Disproportionate or undue burden is the argument wielded to refrain from reasonable accommodation. This term is legislated in different countries on the grounds of criteria (United Nations, 2005; Prideaux, 2006) that can be grouped as follows:

- Costs of accommodation: economic impact and effect on business, reduction of business floor area, cost - benefit analysis of the accommodation, etc.

- Discrimination stemming from failing to implement the measure: the more or less public nature of the business, the estimated number of people with disabilities and usage timeframe, etc.

- Characteristics of the subject required to make accommodation: financial resources, size of company or business, number of employees, the country's economic climate, etc.

- Likelihood of obtaining financing: access to public or private loans, government subsidies, tax benefits, etc.

3. Scope: The scope of the reasonable accommodation notion includes the fields of:

- Employment: An abundant scientific literature has been developed from the US pioneer legislation. In 1995, Burkhauser had already demonstrated the importance of accommodation according to the specific need of the employee with disabilities, as a strategy to prolong the employment. Recent articles, such as Solovieva in 2009 and 2011, focus on assessing solutions through cost-benefit analysis.

- Housing: Under policies for refurbishment, retrofitting and renovation in neighborhoods, projects, such as Del Moral (2015), Lansley (2005), Serrano, (2018), propose tools for diagnosis, control and intervention in accessibility. 
- The provision of public goods and services: Studies such as Kirchner (2008) and Meager (2002) in establishments or Manley (1996), Lewis (2005) and Bromley (2007) in urban environments, assess the real accessibility according to the mandatory requirements and propose solutions.

In summary, these papers propose essentially two strategies for action:

- in private environments (employment and housing), adaptation to people with disabilities shall be made when a person need such specific accommodation to the extend of removing a barrier;

- in public domains (public goods and services), attempts must be made to ensure compliance with the accessibility requirements applicable to the provision of public services, before people with disabilities may require this adjustment.

Regarding their methodological analysis, the purpose is to find factors that influence the adaptation and tools that detect these factors. The difference from previous studies is the factor analysis methodology, which is based here on the legal definition of 'reasonable adjustment'; taking into account that, in public domains, the accommodation to all situations of disability is the objective, as discussed. The criteria showed above, served as a basis for the assessed variables (Section 3.2).

From a regulatory point of view, the complexity of this legal concept, incorporated into the international legislative framework from the adoption of the Convention, has given rise to considerable debate around its interpretation and implementation, as well as has resulted in different responses worldwide (Waddington, 2008). Two different policies for applying reasonable adjustments have been observed (Roulstone, 2009; Cabra de Luna, 2004):

\section{Continental European model. The technical prescription to secure human rights:}

In nations where set procedures have to be followed before a project can be undertaken with a local building permit; the authorisation to commence cannot be given unless the projected works conform to the technical standards. In this case, the main difficulty is to establish what is reasonable in the technical codes.

The following cases exemplify the model:

- In France, for establishments open to the public, a regulatory framework, administrative control, sanctions and financing mechanisms have been articled in order to plan the adjustments (Campion, 2013). Under an administrative procedure (called 'Ad'AP, agenda d'accessibilité programmée'), the accessibility in establishments must be evaluated as defined in specific regulatory provisions, which states the scope and flexibility in compliance. If adaptation is required, works must be executed within a period according to the building typology ( $L O I n^{\circ}$ 2005-102 du 11 février 2005 pour l'égalité des droits et des chances, la participation et la citoyenneté des personnes handicapées).

- In Switzerland, for existing buildings, a 'proportionality' clause has been included, which makes the adaptation mandatory if the works represent less than $20 \%$ of the renovation costs or less than $5 \%$ of the building value. (Loi fédérale du 13 décembre 2002 sur l'élimination des inégalités frappant les personnes handicapées)

- In Spain, for dwellings, the term 'reasonable adjustment' has been defined. The adaptation of communal areas is compulsory when a person with disabilities requires it, and the cost does not exceed a regulated amount. In addition, subsidies are granted to promote this kind of works.

2. Anglo-Saxon or free-market model. Flexibility and reasonableness to avoid discrimination:

By the lack of an officially recognised regulatory body responsible for overseeing such decisions, the test for 'reasonableness' is operated by the service provider and can be challenged by users on an individual basis through the law courts.

In the United Kingdom, one of the most significant examples, the Disability Discrimination Act of 1995, required reasonable adjustments to be made so that people with disabilities are not excluded from workplaces, public buildings and places that serve the public. The deadline for totally meeting that obligation was October 2004. However, Bromley noted in 2007 that the Federation of Small Businesses had reported that $42 \%$ of their members had done nothing to comply with the Act. Once again, the Equality Act of 2010 reinitiated this obligation, making compulsory Codes of Practices (Equality and Human Rights Commission, 2011). The results are still unsatisfactory and a coherent 
and transparent strategy managed by Public Authorities, different from the current model of enforcement that relies on litigation by private individuals, is requested (Women and Equalities Committee, 2017). Other studies remarked this need (Pridaux, 2006; Pérez Bueno, 2012)

The results of this research, close to the first policy model, are intended to constitute an advance in the Spanish regulatory definition of reasonable adjustment, also in establishments.

\section{Methodology.}

\subsection{Sampling and case study}

Previous studies showed accessibility depends largely on urban layout. In 2005 Lewis analysed accessibility in four cities in the United Kingdom. The findings concurred with what was expected: the ascending order of accessibility was a rural town, a monumental city, a large metropolis and the most modern of the four cities studied.

In any given urban nucleus, the most vulnerable quarters tend to be the most scantly adapted, represented by historic centres, spontaneous growth areas and workers' neighbourhoods (Tecnalia, 2011).

In 2007 Bromley noted that although accessibility is lower in city centres, urban improvement policy normally focuses on such areas. Consequently, whereas city centres are gradually turned around into more modern and accessible shopping districts, accessibility shortcomings persist in the suburbs and secondary commercial routes due to vehicle traffic and the prevalence of tradition in local markets.

This survey was conducted in Madrid, a city of a size that ensures the feasibility of representative and varied sampling in a short period of time. Moreover, the choice was also based on the capital city's potential to define trends. With a view to covering a wider variety of solutions, the sample was expanded to include four cities in the metropolitan area (Getafe, Las Rozas, Móstoles and Valdemoro) with a population between 3 million and 70 thousand inhabitants, likewise characterised by the necessary size and regulatory capacity.

The selected case study is representative of city centres, spontaneous growth areas and workers' neighbourhoods, both historic and modern. Additionally, rural areas and cities with a complex topography should be compared.

Focusing on service providers in relation with reasonable adjustments, in 2002 Meager defined a series of factors that favour the adaptation of areas providing services to the public in United Kingdom: large scale (75\% adapted), membership in a large organisation (50\% adapted), government ownership and voluntary sector establishments (60\% adapted); existence of people with disabilities on the payroll; and use of facilities by customers with disabilities.

Conversely, accessibility shortcomings are primarily found in neighbourhood markets, shops, bars and restaurants, the main sources of community services, in contrast to state/institutional and health carerelated establishments (Lewis, 2005; Prost-Coletta, 2015).

In the Spanish case, although the least frequent breaches of the legislation in buildings involve entrances, inter-storey vertical circulation and toilets (Fundación ONCE, 2013), they are the object of the largest number of claims (Figure 1). Even today, the lack of adaptation constitutes an insurmountable barrier for the mobility of wheelchair users. 


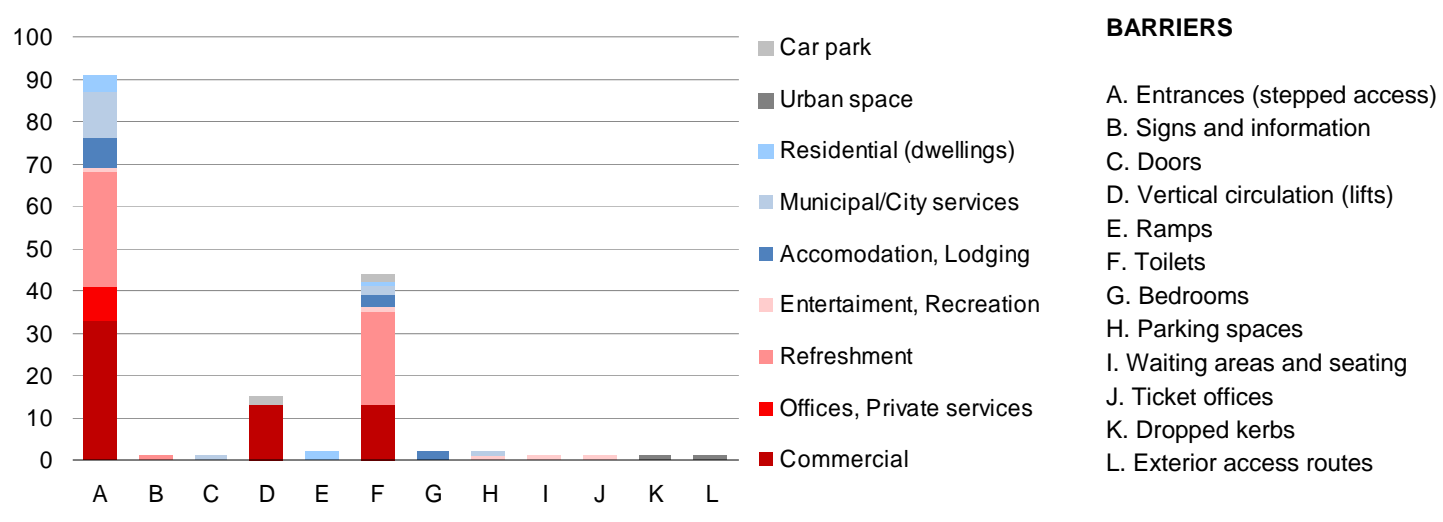

Figure 1. Number of accessibility complaints in the region of Madrid by activity and barrier (2007-2013). Source: Madrid Regional Council for the Furtherance of Accessibility and Removal of Barriers (Consejo para la Promoción de la Accesibilidad y Supresión de Barreras de la Comunidad de Madrid).

In Madrid region, small establishments account for most of the built area open to the public. According to its Regional Statistics Institute, in 2010 only $17 \%$ of the region's establishments had over 10 employees (Madrid, 2011).

Moreover, business owners' limited yearly capacity to remodel their premises $(0.5 \%$ according to the statistics on business premises authorisations issued by the City of Madrid in 2014), together with the difficult task of controlling the results, given the lack of legislative tools on adaptations available to the authorities, translate into a pace of change that has been too slow to meet the 4 December 2017 deadline, in need of a complete strategy.

For that purpose, a total of 120 administrative dossiers furnished by the respective city governments were assessed, involving interventions meeting the following criteria:

- small establishments with a net floor area of up to $200 \mathrm{~m}^{2}$ in existing buildings, spaces more difficult to adapt and with the most of the built area open to the public, as mentioned earlier;

- administrative dossier finalising in 2013 or 2014, i.e., with the most recent accessibility solutions;

- ground floors and professional offices in above-grade storeys, the two types of usual establishments found in the city's neighbourhoods;

- works involving difficulty comparable to accessibility improvements, such as extensions to premises, changes of use, changes in business activity, functional upgrading or refurbishment, excluding new construction.

\subsection{Analytical variables}

As discussed in Section 2, the variables addressed were grouped into families (Figure 2) on the grounds of the legal concept of 'reasonable accommodation' to define the scope of that concept.

By way of summary, 21 variables describing establishments, 20 relating to proportionality and 30 to element (NAe) and overall (NAg) accessibility categories were analysed (the paper only shows the most significant results and variables). 


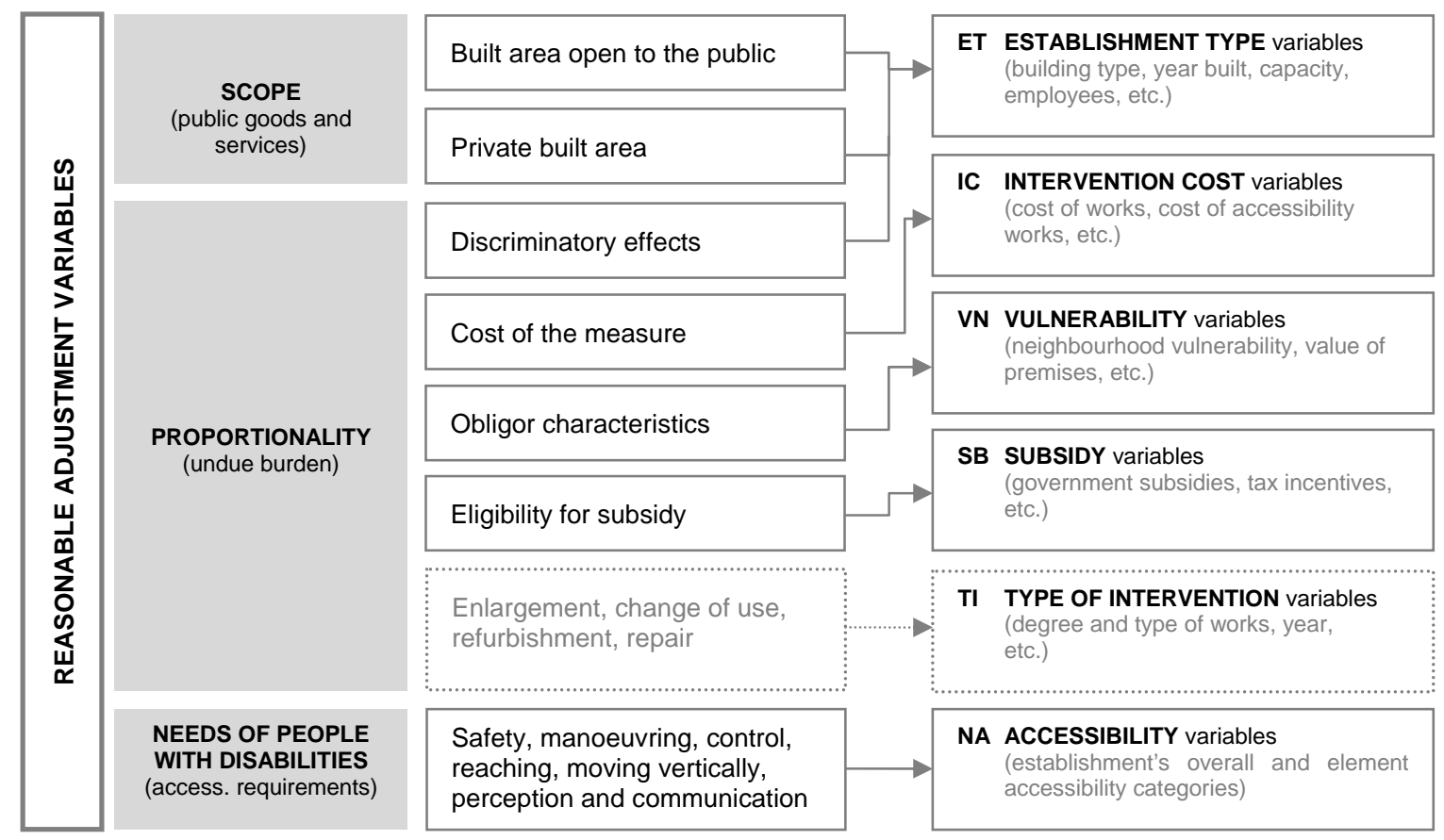

Figure 2. Design criteria for 'reasonable adjustment' variables

\subsection{Accessibility requirements to be met by buildings}

Table 1 shows an evaluation of the technical difficulties and costs of different measures adopted to fulfil accessibility requirements. It reveals that the requirements relating to manoeuvre and to navigate vertically (in bold lettering) pose substantial technical problems and involve considerable expense in interventions on existing buildings.

Actions that entail structural modifications are logically complex and costly. At the same time, the smaller the establishment, the greater the functional impact of the loss of floor area inherent in accommodating accessible elements.

Measures that primarily impact wheelchair users' mobility, i.e., to manoeuvre and to navigate vertically, are the ones that are most difficult to implement and that call for regulatory flexibility (Table 2). The others can be easily met as required in new buildings.

Table 1. Impact-effectiveness assessment of accessibility in buildings

\begin{tabular}{|c|c|c|c|c|c|c|}
\hline Difficulty & $\begin{array}{l}\text { Feature } \\
\text { involved }\end{array}$ & $\begin{array}{l}\text { Measure to be } \\
\text { adopted }\end{array}$ & User $^{(1)}$ & $\begin{array}{l}\text { Absence of the } \\
\text { measure } \\
\text { entails }\end{array}$ & $\begin{array}{l}\text { Technical } \\
\text { difficulty } \\
\text { involved }\end{array}$ & $\begin{array}{c}\text { Cost of the } \\
\text { measure }\end{array}$ \\
\hline \multirow{3}{*}{$\begin{array}{l}\text { To detect } \\
\text { obstacles and } \\
\text { hazards }\end{array}$} & \multirow{2}{*}{$\begin{array}{l}\text { Protection from } \\
\text { falling }\end{array}$} & Signs & VIP & Unsafe use & --- & Very low \\
\hline & & Barriers & $\begin{array}{l}\text { Children } \\
\text { VIP }\end{array}$ & Unsafe use & --- & Intermediate \\
\hline & $\begin{array}{l}\text { Prevention of } \\
\text { collision with } \\
\text { unperceived } \\
\text { elements }\end{array}$ & $\begin{array}{l}\text { Glass surface } \\
\text { warnings }\end{array}$ & VIP & Unsafe use & --- & Very low \\
\hline \multirow{2}{*}{ To manoeuvre } & \multirow{2}{*}{$\begin{array}{l}\text { Horizontal } \\
\text { circulation }\end{array}$} & Clear width & $\begin{array}{l}\text { WCU } \\
\text { PRM }\end{array}$ & Hampered use & $\begin{array}{c}\text { Dimensional } \\
\text { Structural }\end{array}$ & Very high \\
\hline & & Turning & $\begin{array}{l}\text { WCU } \\
\text { PRM }\end{array}$ & Hampered use & $\begin{array}{c}\text { Dimensional } \\
\text { Structural }\end{array}$ & Very high \\
\hline \multirow{3}{*}{$\begin{array}{l}\text { To navigate } \\
\text { vertically }\end{array}$} & \multirow{3}{*}{$\begin{array}{l}\text { Vertical } \\
\text { circulation }\end{array}$} & & WCU & & Dimensional & \\
\hline & & Ramps & PRM & Hampered use & Structural & Very high \\
\hline & & Lifts & $\begin{array}{l}\text { WCU } \\
\text { PRM }\end{array}$ & Hampered use & $\begin{array}{c}\text { Dimensional } \\
\text { Structural }\end{array}$ & Very high \\
\hline $\begin{array}{c}\text { To exercise } \\
\text { control }\end{array}$ & Ancillary elements & $\begin{array}{l}\text { Design of door } \\
\text { handles, switches, } \\
\text { call buttons, etc. }\end{array}$ & PID & Difficult use & --- & Low \\
\hline
\end{tabular}




\begin{tabular}{|c|c|c|c|c|c|c|}
\hline Difficulty & $\begin{array}{l}\text { Feature } \\
\text { involved }\end{array}$ & $\begin{array}{l}\text { Measure to be } \\
\text { adopted }\end{array}$ & User $^{(1)}$ & $\begin{array}{l}\text { Absence of the } \\
\text { measure } \\
\text { entails }\end{array}$ & $\begin{array}{l}\text { Technical } \\
\text { difficulty } \\
\text { involved }\end{array}$ & $\begin{array}{c}\text { Cost of the } \\
\text { measure }\end{array}$ \\
\hline & & Door opening force & $\begin{array}{l}\text { WCU } \\
\text { PRM } \\
\text { PIS } \\
\text { PID }\end{array}$ & Difficult use & --- & Low \\
\hline To reach & Ancillary elements & $\begin{array}{l}\text { Door handles, } \\
\text { switches, intercoms, } \\
\text { etc. }\end{array}$ & $\begin{array}{l}\text { WCU } \\
\text { PHI }\end{array}$ & Difficult use & --- & Low \\
\hline $\begin{array}{l}\text { To perceive } \\
\text { and } \\
\text { communicate }\end{array}$ & Building layout & $\begin{array}{l}\text { Information and } \\
\text { signs }\end{array}$ & $\begin{array}{l}\text { VIP } \\
\text { HIP } \\
\text { SIP } \\
\text { CIP }\end{array}$ & Difficult use & --- & Low \\
\hline
\end{tabular}

(1) Type of user with special protection on the grounds of age or disability: WCU- wheelchair users; PRM- people with reduced mobility; PHI- people with height-related impairments; PIS- people with impaired strength; PID- people with impaired dexterity; VIP- visually impaired people; HIP- hearing impaired people; SIP- speech impaired people; CIP-cognitive impaired people

\subsubsection{Element accessibility variables (NAe)}

To quantify such flexibility, accessibility categories and its value were defined further to the criteria set out below.

- Accessible (score of 3): the adapted elements meet functional and dimensional requirements laid down in the Spanish Building Code (Real Decreto 173/2010).

- Practicable (score of 2): although the adapted elements fail to conform to legal requirements, some wheelchair users can access and use the premises more or less autonomously. This category concurs with the basic accessibility conditions defined in the regional and national regulations in effect prior to enactment of the building code and with the provisions of international regulations in existing buildings (ISO 21542:2012; Alegre, 2005).

- Practicable with assistance (score of 1): barriers render the adapted spaces wheelchair-accessible only with assistance. This category is defined on the grounds of the solutions found in the dossiers assessed.

- Non-wheelchair accessible (score of 0): none of the above requisites is met.

The requirements defining the accessibility categories by variable of horizontal and vertical circulation elements (NAe) are given in Table 2. This variables are detailed in Figure 3.

Table 2. Accessibility category requirements (summarized)

\begin{tabular}{|c|c|c|c|c|}
\hline Difficulty & Variable $N A e$ & Accessible (3) & Practicable (2) & $\begin{array}{l}\text { Practicable with } \\
\text { assistance (1) }\end{array}$ \\
\hline \multirow{7}{*}{ To manoeuvre } & Clear width & $1.20 \mathrm{~m}$ & $0.90 \mathrm{~m}$ & --- \\
\hline & Occasional narrowing & $1.00 \mathrm{~m}$ to $<50 \mathrm{~cm}$ depth & $0.80 \mathrm{~m}$ & $0.80 \mathrm{~m}$ \\
\hline & $>90^{\circ}$ turn & $\varnothing 1.50 \mathrm{~m}$ & $\varnothing 1.20 \mathrm{~m}$ & $\varnothing 1.50 \mathrm{~m}$ \\
\hline & Transfer space & $0.80 \mathrm{~m}$ on both sides & $0.80 \mathrm{~m}$ on one side & --- \\
\hline & $\begin{array}{l}\text { Clear width through a } \\
\text { doorway }\end{array}$ & $0.80 \mathrm{~m}$ (at least one leaf) & $0.75 \mathrm{~m}$ (at least one leaf) & $\begin{array}{c}0.80 \mathrm{~m} \text { (total clearance, } \\
\text { although the leaves are } \\
\text { narrower) }\end{array}$ \\
\hline & $\begin{array}{l}\text { Distance from handle to } \\
\text { corner }\end{array}$ & $30 \mathrm{~cm}$ & --- & 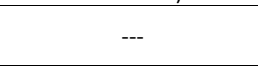 \\
\hline & Door opening force & $<25 \mathrm{~N}$ & --- & --- \\
\hline \multirow[t]{5}{*}{$\begin{array}{l}\text { To navigate } \\
\text { vertically }\end{array}$} & Vertical circulation & $\begin{array}{c}\text { Accessible lift } \\
\text { Accessible ramp }\end{array}$ & $\begin{array}{c}\text { Practicable lift } \\
\text { Practicable ramp } \\
\text { Vertical lifting platform } \\
\text { Wheelchair platform } \\
\text { stairlift }\end{array}$ & $\begin{array}{c}\text { Step }<20 \mathrm{~cm} \\
\text { Seated stairlift } \\
\text { Ramp practicable with } \\
\text { assistance }\end{array}$ \\
\hline & Ramps, slope & $\begin{array}{l}10 \% \text { up to } 3 \mathrm{~m} \\
8 \% \text { up to } 6 \mathrm{~m} \\
6 \% \text { up to } 9 \mathrm{~m}\end{array}$ & $\begin{array}{l}12 \% \text { up to } 3 \mathrm{~m} \\
10 \% \text { up to } 10 \mathrm{~m} \\
8 \% \text { up to } 15 \mathrm{~m} \\
6 \% \text {, unlimited }\end{array}$ & $<16 \%$ up to $1.25 \mathrm{~m}$ \\
\hline & $\begin{array}{l}\text { Ramps, intermediate } \\
\text { landings }\end{array}$ & $1.50 \mathrm{~m}$ & $1.20 \mathrm{~m}$ & --- \\
\hline & $\begin{array}{l}\text { Ramps, landings in front } \\
\text { of doors }\end{array}$ & $1.50 \mathrm{~m}$ & $1.20 \mathrm{~m}$ & --- \\
\hline & $\begin{array}{l}\text { Ramps, without landings } \\
\text { in front of doors }\end{array}$ & $\begin{array}{r}\text { If door is } \\
\text { (in outages, } \mathrm{m}\end{array}$ & $\begin{array}{l}\text { Atomatic } \\
\text { remain open) }\end{array}$ & $\begin{array}{l}\text { If call button is accessible } \\
\text { at bottom of ramp }\end{array}$ \\
\hline
\end{tabular}




\begin{tabular}{llccc}
\hline Difficulty & Variable NAe & Accessible (3) & Practicable (2) & $\begin{array}{c}\text { Practicable with } \\
\text { assistance (1) }\end{array}$ \\
\hline & $\begin{array}{l}\text { Lift } \\
\text { (width } \mathrm{x} \text { depth) }\end{array}$ & $\begin{array}{c}1.00 \mathrm{~m} \times 1.25 \mathrm{~m} \\
\text { (one entrance, same side } \\
\text { on all storeys) }\end{array}$ & $\begin{array}{c}0.90 \mathrm{~m} \times 1.20 \mathrm{~m} \\
\text { (one entrance, same side } \\
\text { on all storeys) }\end{array}$ & --- \\
\hline
\end{tabular}

\subsubsection{Overall accessibility variables (NAg)}

In addition to the above, variables were formulated to quantify overall establishment accessibility. The following $N A g$ variables were the most significant for the study:

- NAg_pub: overall accessibility in areas open to the public (Table 3): this index was calculated bearing in mind that an inaccessible element renders the downstream chain inaccessible (any element located after a point that is not surmountable with a wheelchair is considered inaccessible, 0 score, regardless of its individual element accessibility). It is the arithmetic average of the NAe values of the elements intended for public use of each establishment.

$$
N A g_{-} p u b=\frac{1}{n} \sum_{i=1}^{n} N A e_{i}
$$

Table 3. Example of calculation for 8 establishments in Madrid (Nag_pub)

\begin{tabular}{|c|c|c|c|c|c|c|c|c|c|c|c|c|c|c|c|c|c|}
\hline 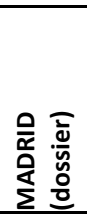 & 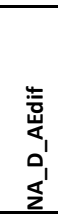 & 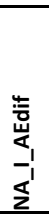 & 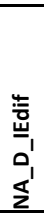 & 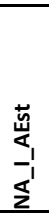 & $\begin{array}{l}0 \\
\frac{0}{2} \\
-1 \\
\frac{1}{z}\end{array}$ & $\begin{array}{l}\frac{0}{z} \\
\frac{2}{1} \\
\frac{n}{5} \\
\frac{\pi}{z}\end{array}$ & $\begin{array}{l}z_{1} \\
u_{1} \\
z_{1} \\
\frac{1}{z}\end{array}$ & 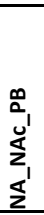 & $\begin{array}{l}0 \\
0_{1}^{\prime} \\
u^{\prime} \\
\Sigma_{1} \\
\Sigma_{1}\end{array}$ & $\begin{array}{l}0 \\
0 \\
0 \\
\vdots \\
\frac{1}{1} \\
-1 \\
\frac{1}{z}\end{array}$ & 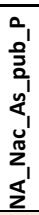 & $\begin{array}{l}0 \\
0 \\
0 \\
\Sigma_{1} \\
z\end{array}$ & $\begin{array}{l}0 \\
0 \\
0 \\
2 \\
0 \\
-1 \\
1 \\
z \\
z\end{array}$ & 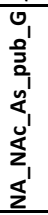 & $\begin{array}{l}\bar{\Xi} \\
\underline{\Xi} \\
\end{array}$ & $\begin{array}{l}\text { E } \\
\text { Dે } \\
\text { U્ }\end{array}$ & 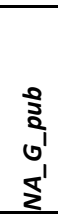 \\
\hline M01 & 1 & 2 & & & 1 & & & & 0 & 0 & 0 & 0 & 0 & & 4 & 8 & 0.50 \\
\hline M02 & 1 & 2 & & & 3 & & & & & & & & & & 6 & 3 & 2.00 \\
\hline M03 & & 2 & & & 3 & & & 0 & 0 & & & & & & 5 & 4 & 1.25 \\
\hline M04 & & 0 & & & 0 & & & & & & & & & & 0 & 2 & 0.00 \\
\hline M05 & 1 & 2 & & & 3 & & & & & & & 0 & & & 6 & 4 & 1.50 \\
\hline M06 & 1 & 2 & & & 3 & 0 & & & & & & 0 & & & 6 & 5 & 1.20 \\
\hline M07 & 0 & 0 & 0 & 0 & 0 & 0 & & & & & & & & & 0 & 6 & 0.00 \\
\hline M08 & 1 & 2 & & & 3 & & & 0 & & & & 0 & & & 6 & 5 & 1.20 \\
\hline 0,0 & Inac & & & & & & & & & & & & & & & & \\
\hline 0,0 & Non & cess & & hent & & $n$ & & & & & & & & & & & \\
\hline
\end{tabular}

- NAg_change: overall accessibility after the changes made in the premises (Table 4): it is the arithmetic average of the difference between the initial and final NAe values for each element of each establishment.

$$
N A g_{-} \text {change }=\sum_{i=1}^{n}\left(N A \text { e. } \text { final }_{i}-\text { NAe.initial }_{i}\right)
$$

\begin{tabular}{|c|c|c|c|c|c|c|c|c|c|c|c|c|c|c|c|c|c|c|c|c|c|c|c|c|c|c|c|c|c|c|c|c|c|c|c|c|c|}
\hline 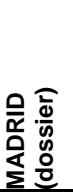 & 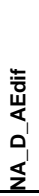 & 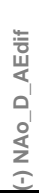 & 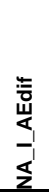 & 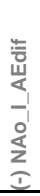 & $\begin{array}{l}0 \\
\underline{z} \\
\vdots \\
\vdots \\
\Sigma\end{array}$ & 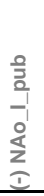 & $\begin{array}{l}\geq \\
\frac{2}{2} \\
\vdots \\
\Sigma\end{array}$ & $\begin{array}{l}\geq \\
\geq \\
\frac{2}{2} \\
0 \\
0 \\
\frac{1}{2} \\
\frac{1}{1} \\
\end{array}$ & 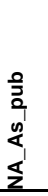 & 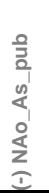 & 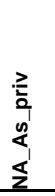 & 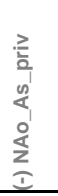 & $\begin{array}{l}7 \\
\& \\
\mathbb{8} \\
z\end{array}$ & $\begin{array}{l}\frac{Z}{<} \\
0 \\
\frac{1}{1} \\
z \\
\end{array}$ & $\begin{array}{l}\infty \\
\underline{a} \\
\Sigma\end{array}$ & $\begin{array}{l}m \\
\frac{m}{2} \\
0 \\
\frac{1}{2} \\
z \\
\end{array}$ & $\begin{array}{l}0 \\
0 \\
\Sigma \\
z\end{array}$ & $\begin{array}{l}0 \\
0 \\
0 \\
0 \\
\frac{1}{2} \\
z \\
1 \\
\end{array}$ & $\begin{array}{l}a \\
a \\
0 \\
a \\
\vdots \\
\vdots \\
z \\
z\end{array}$ & $\begin{array}{c}0 \\
0 \\
0 \\
z \\
-1 \\
0 \\
0 \\
\frac{1}{z} \\
\frac{1}{2}\end{array}$ & 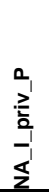 & 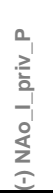 & 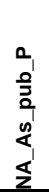 & 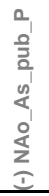 & 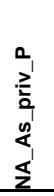 & 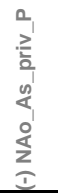 & $\begin{array}{l}0 \\
0 \\
\vdots \\
\Sigma\end{array}$ & $\begin{array}{l}0 \\
0 \\
0 \\
0 \\
\frac{1}{2} \\
1 \\
\end{array}$ & $\begin{array}{l}0 \\
\vdots \\
0 \\
\vdots \\
\vdots \\
\Sigma \\
\Sigma\end{array}$ & 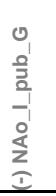 & $\begin{array}{l}0 \\
\geq \\
\geq \\
2 \\
-1 \\
\Sigma \\
\end{array}$ & 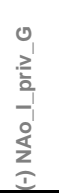 & 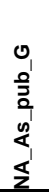 & 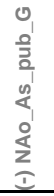 & $\begin{array}{l}0 \\
\geq \\
\geq \\
\vdots \\
0 \\
0 \\
\vdots \\
\Sigma \\
z\end{array}$ & 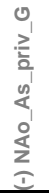 & $\begin{array}{l}0 \\
\vdots \\
3 \\
0 \\
0 \\
\vdots \\
\vdots\end{array}$ \\
\hline M01 & 1 & -1 & 2 & -2 & 1 & -3 & 0 & -0 & & & & & & & & & 0 & -0 & 0 & -0 & 0 & -0 & 0 & & 0 & -0 & 0 & -0 & 3 & -3 & 0 & -0 & & & 0 & -0 & -2 \\
\hline M02 & 1 & -1 & 2 & -2 & 3 & -3 & 0 & -0 & & & 0 & -0 & & & & & & & & & & & & & & & & & & & & & & & & & 0 \\
\hline M03 & & & 2 & -2 & 3 & -3 & & & & & & & & & 0 & & 0 & -0 & & & 0 & -0 & & & 0 & -0 & & & & & & & & & & & 0 \\
\hline M04 & & & 2 & -2 & 3 & -3 & 0 & -0 & & & 0 & -0 & & & & & & & & & & & & & & & & & & & & & & & & & 0 \\
\hline M05 & 1 & -1 & 2 & -2 & 3 & -3 & 0 & -0 & & & & & & & & & & & & & & & & & & & 0 & -0 & & & 0 & -0 & & & 0 & -0 & 1 \\
\hline M06 & 1 & -1 & 2 & -2 & 3 & -3 & 0 & -0 & 0 & -0 & & & & & & & & & & & & & & & & & 0 & -0 & & & 3 & -0 & & & 0 & -0 & 3 \\
\hline M07 & 0 & -0 & 2 & -2 & 0 & -0 & & & 0 & & & -0 & & & & & & & & & & & & & & & & & & & & & & & & & 0 \\
\hline M08 & 1 & -1 & 2 & -2 & 3 & -3 & & & & & & & & & 0 & & & & & & & & & & & & 0 & -0 & & & 3 & -3 & & & 0 & -0 & 0 \\
\hline
\end{tabular}

Table 4. Example of calculation for 8 establishments in Madrid (NAg_change) 


\subsection{Statistical analysis}

Because no assumption of 'normality and homogeneity of variance' can be met, this study makes use of 'nonparametric statistics'. The specific nonparametric test is detailed in each section.

Additionally, 'robust statistics' has been used because they are statistics with good performance for data drawn from a wide range of probability distributions, especially for distributions that are not normal. One motivation is to produce statistical methods that are not unduly affected by outliers. The 'median', a robust measure of central tendency, unlike the 'mean'; and 'interquartile range', a robust measure of statistical dispersion, were used consequently in this study. 'Box plot' graph appropriately these parameters.

\section{Results and discussion}

\subsection{Characterisation of accessibility solutions (NAe)}

The frequency (in percentage) of the accessibility category (accessible, practicable, practicable with assistance, inaccessible) for each (NAe) element in the establishment analysed is graphed in Figure 3. The figure includes a characteristic cross-section of the establishments, specifying whether they were at (premises 1) or above (premises 2) street level, for elements in areas both open to the public (lettered '_pub') and for the exclusive use of employees (lettered '_priv'), and whether the elements were downstream of slightly stepped accesses ('_P') or of full storey staircases ('_G').

The elements positioned below the dotted red line, while potentially accessible, are shown as inaccessible and their accessibility category is changed to ' $O$ ' (see Table 3 ) because they were located downstream of an inaccessible element, resulting in a missed opportunity to improve accessibility.

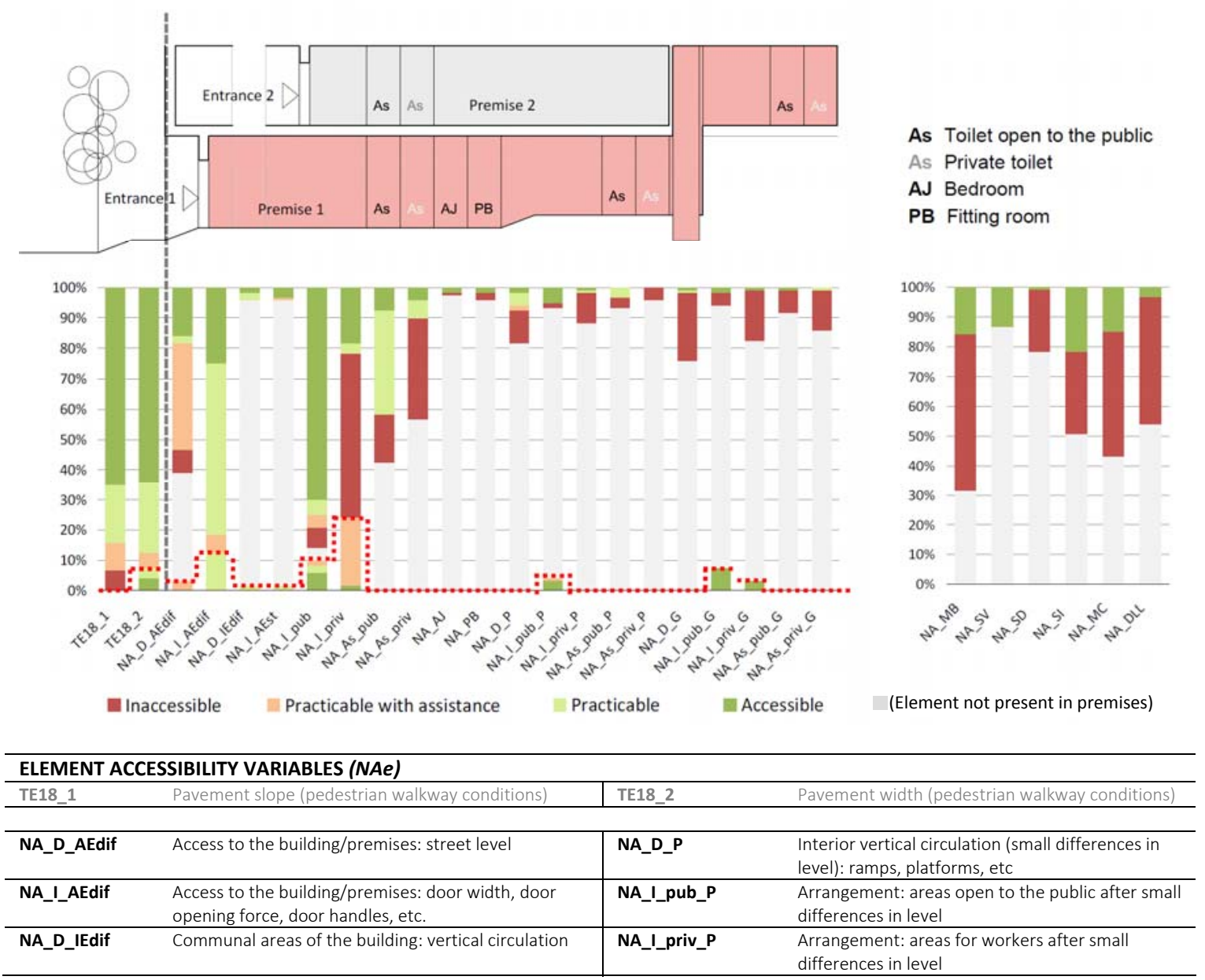




\begin{tabular}{ll|ll}
\hline ELEMENT ACCESIBILITY VARIABLES (NAe) & Communal areas of the building: access to the \\
\hline NA_I_AEst & premises on first floors & NA_As_pub_P & $\begin{array}{l}\text { Toilet open for customers after small differences } \\
\text { in level }\end{array}$ \\
\hline NA_I_pub & Arrangement: areas open to the public & NA_As_priv_P & Toilet for workers after small differences in level \\
\hline NA_I_priv & Arrangement: areas for workers & $\begin{array}{l}\text { Interior vertical circulation (one floor or more): } \\
\text { lift }\end{array}$ \\
\hline NA_As_pub & Toilet for customers & NA_I_pub_G & $\begin{array}{l}\text { Arrangement: areas open to the public on floors } \\
\text { other than the ground one }\end{array}$ \\
\hline NA_As_priv & Toilet for workers & NA_I_priv_G & $\begin{array}{l}\text { Arrangement: areas for workers on floors other } \\
\text { than the ground one }\end{array}$ \\
\hline NA_AJ & Bedroom (lodging) & NA_As_pub_G & $\begin{array}{l}\text { Toilet for customers on floors other than the } \\
\text { ground one }\end{array}$ \\
\hline NA_PB & Fitting room (commercial) & NA_As_priv_G & $\begin{array}{l}\text { Toilet for workers on floors other than the } \\
\text { ground one }\end{array}$ \\
\hline \multicolumn{2}{l}{} & NA_SI & Signs and information of accessibility \\
\hline NA_MB & Customer service counters or windows & NA_MC & Switches \\
\hline NA_SV & Contrast bands on glass surfaces & NA_DLL & Call buttons in toilets for customers \\
\hline
\end{tabular}

Figure 3. Accessibility chain: actual and potential post-intervention accessibility category by element

Adaptation of an establishment's entrance relative to street level (variable NA_D_AEdif) was the first and consequently most critical element in the accessibility sequence. Conversely, in the cases analysed, access was not prevented by other accessibility conditions required to the entrance (grouped in variable NA_I_AEdif).

In addition to the entrances, the elements most frequently remodelled in these premises were the areas open to the public on ground floors (variable NA_I_pub: $95 \%$ present in the dossiers), in which adaptation was successful to some degree of accessibility in $80 \%$ of the cases. The aim was to provide all services or at least one of each type of service to the public in these areas, a basic recommendation established by best practice accessibility manuals in complex adaptations (Disability Right Commission, 2005).

Public toilets were another of the most frequently modified elements (variable NA_As_pub: $58 \%$ present in the dossiers), with a total of $42 \%$ successfully adapted on the ground or access storey. Toilets share with accesses the drawback that adaptations may have a significant impact on the occupied net floor area of the premises or a scantly modifiable structural element. These two elements (entrances and toilets), object of frequent demands (Figure 1), are consequently analysed in greater detail in Sections 4.1.1 and 4.1.2. However, lifts lacked a representative sample to be analyzed (NA_D_G) due to the size of premises.

Although the areas for the exclusive use of employees were not the specific matter of this analysis, in the dossiers analysed, most of them were not adapted (their size exempted them from building code regulations). The successful accessibility scores for these spaces were due to their open plan layout or to previous adaptations made to conduct a different business. As explained in Section 2, adaptation must be subject to reasonable adjustments in employment.

Where inaccessible, neither the street conditions (TE18_1 and TE18_2) nor the communal areas inside the building (NA_D_IEdif and NA_I_AEst) could be modified in the remodels studied, inasmuch as they did not belong exclusively to the owner of the premises.

Scant references were made in the projects to other accessibility elements required by the legislation (shown in green on the bar graph on the right), such as contrast bands on glass surfaces (NA_SV), staircase warning signs (NA_SD), signs and information (NA_SI), accessible customer service counters or windows (NA_MB), light switches (NA_MC) or call buttons in public toilets (NA_DLL). Lewis in 2005 also warned that establishments presented a low level of accessibility more pronounced in the aspects related to cognitive and sensory disabilities, on which there is a low level of awareness. On the other hand, as analysed in Section 3.3, the adaptation is quite easy and inexpensive.

Design explanations in municipal dossiers focused on the wheelchair accessibility sequence in areas open to the public, a finding consistent with the results of other accessibility studies (Section 3.1). 


\subsubsection{Characterisation of entrances (NA_D_AEdif)}

The improvements stemming from the solutions most frequently adopted in the establishments studied (accesses and public toilets) were characterised by accessibility category (Table 5) based on an analysis of the technical solutions observed in the dossiers, and by the most statistically significant variables denoting the degree to which one or another solution was possible.

Table 5. Statistically significant median values of variables and technical solutions of accessibility categories in entrances (variable NA_D_AEdif)

ENTRANCE Median

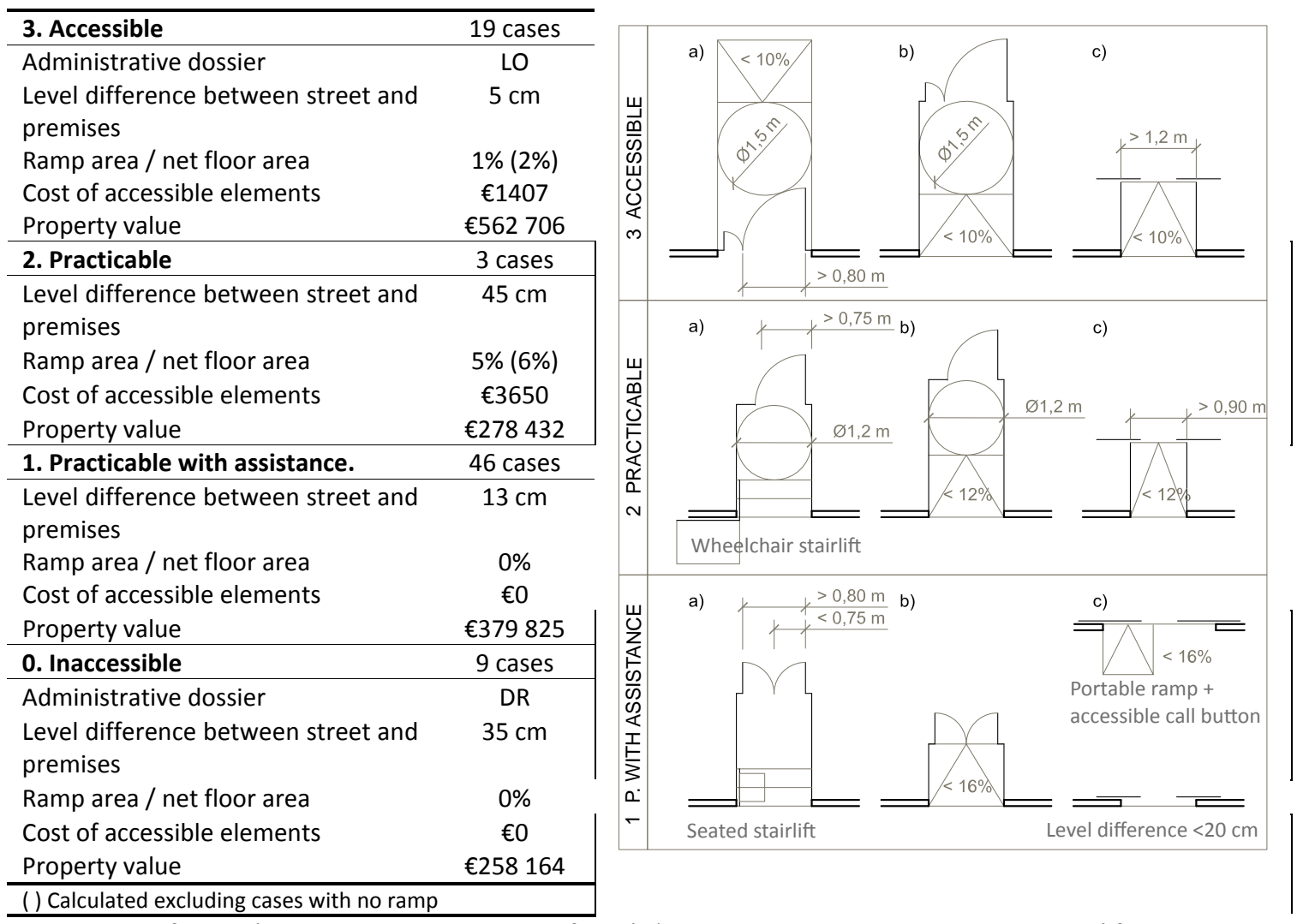

Statistical significance (running by SPSS Statistic solfware): 'Contingency Tables and Chi-Square Test' for categorical variables; 'Kruskal-Wallis H Test' for quantitative ones. Scope and proportionality variables (Figure 2) were assessed. Nonparametric tests.

For entrances, Table 5 shows that the best accessibility results were obtained for dossiers subject to the strictest administrative proceedings $(\mathrm{LO})^{1}$ and where the property value was highest. The (median) height over street level was barely $5 \mathrm{~cm}$, remediable with ramps that barely occupied the net floor area (1\%$2 \%)$.

The cost of full accessibility, around $€ 1400$, was much lower than the $€ 3600$ required to the practicable category, where stairlifts were often used as a solution for (median) height differences of up to $45 \mathrm{~cm}$.

In practicable with assistance solutions, the median accessibility expense was $€ 0$, for the option of choice in most cases was to refrain from modifying entrances stepped by less than $20 \mathrm{~cm}$.

In most of the dossiers analysed, ramps without landings were the solutions of choice, for they forfeited less area than with entrance landings (solutions a) and b) in the figure in Table 5). Such solutions, without entrance landings, were scored as less accessible, for wheelchair users would require assistance to hold the door open during the ramp use. This could be improved by installing an automatic (sliding) door (c)

\footnotetext{
${ }^{1}$ The difference between the most (LO) and the least (DR) strict construction permits is the administrative proceeding. In the former, works cannot be initiated until the design documents are reviewed and approved by the municipal and control authorities, whereas in DR permit, responsibility for compliance is vested in the designer and municipal inspection is conducted with the works underway or finalised, or after the premises are in use.
} 
solution in the figure in Table 5), which would make a horizontal entrance landing unnecessary. Such landings would be needed to enable wheelchair users to independently open manual doors.

The maximum allowable slope on ramps was set at $16 \%$. In these ramps so steeply sloped wheelchair users might also require assistance. Ramps with a higher grade could be hazardous, as observed in an entrance adaptation where a ramp sloped at $21 \%$ was ultimately removed for that reason.

Another extreme solution used to adapt entrances consisted in the use of portable ramps (1c) in the figure in Table 5). Such arrangements require an accessible call button at the entrance to ask for the portable element to be set in place.

Entrances steeped by up to $20 \mathrm{~cm}$ were allowed on the assumption that they can be navigated by wheelchair users with assistance (1c) in the figure in Table 5). One of the establishments reviewed had adapted indoor premises and toilets, even though its entrance was by $23 \mathrm{~cm}$.

Seated and wheelchair stairlifts (1a) and 2a) in the figure in Table 5) were not often used for scantly stepped entrances in light of their high installation and maintenance costs.

\subsubsection{Characterisation of public toilets (NA_As_pub)}

Public toilets followed much the same pattern as entrances, in which the most accessible adaptations were observed in remodels with dossiers subject to the most demanding administrative proceedings (LO), the highest property value (€670 000 median) and highest cost (€2 600 median). All these values declined with the accessibility category.

Table 6. Statistically significant median values of variables and technical solutions of accessibility categories in public toilets (variable NA_As_pub).

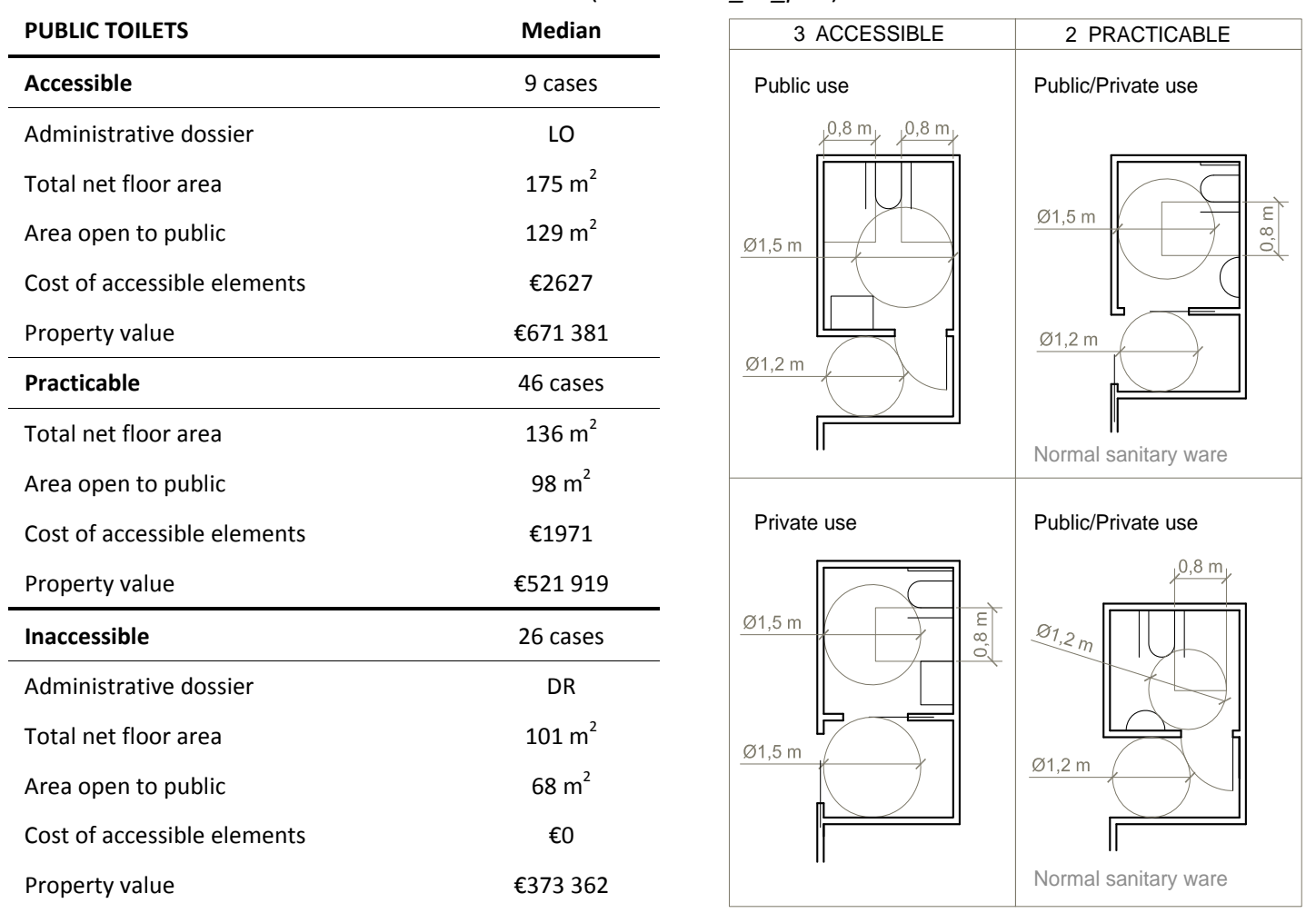

Statistical significance (running by SPSS Statistic solfware): 'Contingency Tables and Chi-Square Test' for categorical variables; 'Kruskal-Wallis H Test' for quantitative ones. Scope and proportionality variables (Figure 2) were assessed. Nonparametric tests.

Premises with a median floor area open to the public of just $68 \mathrm{~m}^{2}$ in a total net floor area of $100 \mathrm{~m}^{2}$ tended to have inaccessible toilets. The optimal results were observed for areas open to the public of around $130 \mathrm{~m}^{2}$ in a total net floor area of $175 \mathrm{~m}^{2}$ (median values). 
Whilst the size of the toilets could have accommodated the compliance of the building code in most of the cases analysed, they were assigned a lower accessibility score due to design errors involving element positions or the use of normal sanitary ware. Even today, accessibility training for technicians and builders is obviously necessary to carry out such adaptations. This observation was also noted by Froehlich-Grobe in 2008.

\subsection{Characterisation of establishments (cost variables)}

Accessibility improvements were assessed in terms of the economic viability of the activity involved. The activities were grouped by type of area (public and private) and accessibility elements, resulting in 13 classes for this study (Figure 4).

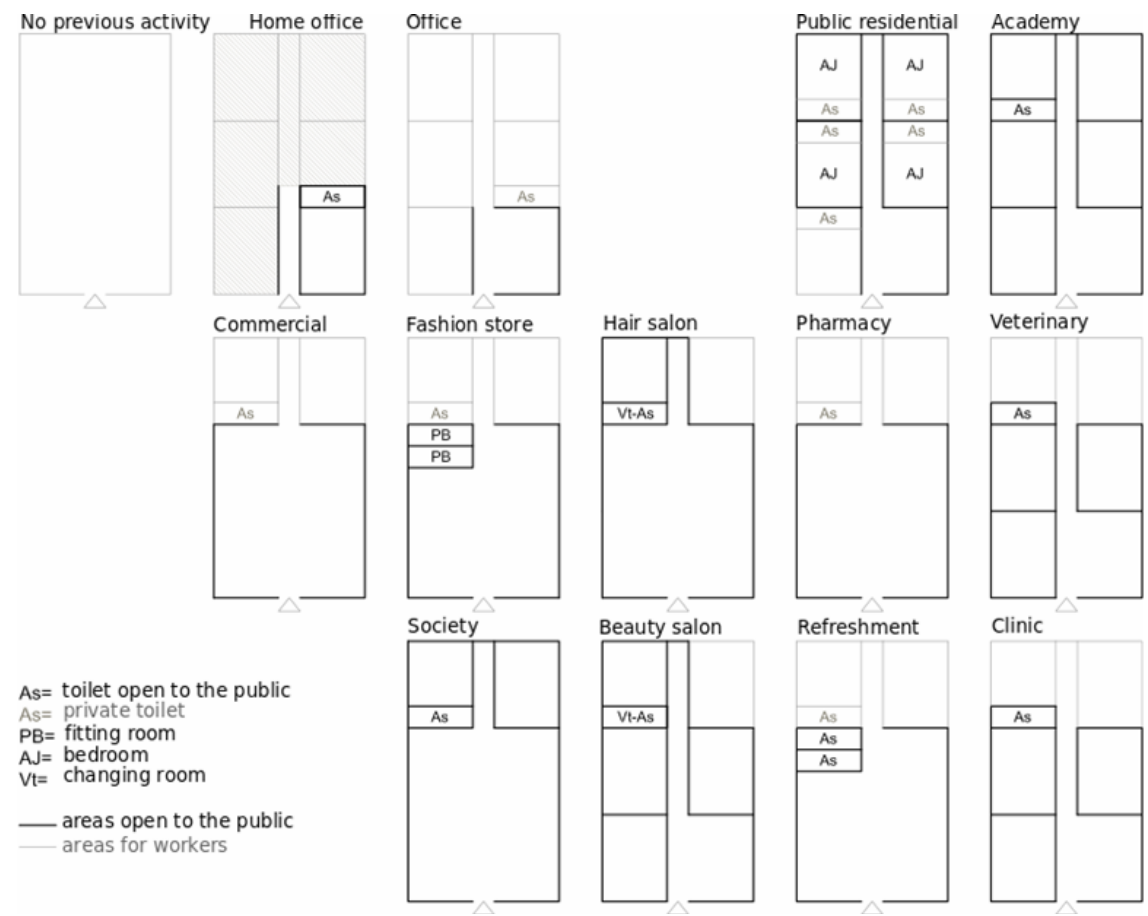

Figure 4. Areas and accessibility elements by activity (simplified plants)

The results (Table 7 and 8), consistent with previous studies in Section 3.1, go in depth in problems of each activity. Certain types of premises, such as clinics, pharmacies and bank branches, proved to be more prone to remodels and therefore attained higher accessibility scores. The works also tended to be subject to more demanding administrative requirements. Retail businesses, hair and beauty parlours and academies, which generally occupy premises pre-adapted to their needs, were less likely to remodel. Their accessibility scores were normally based on their original status related to the street level 
Table 7: Economic impact of accessibility improvements on activity

(Box plot, median and interquartile rage, running by SPSS Statistic software)

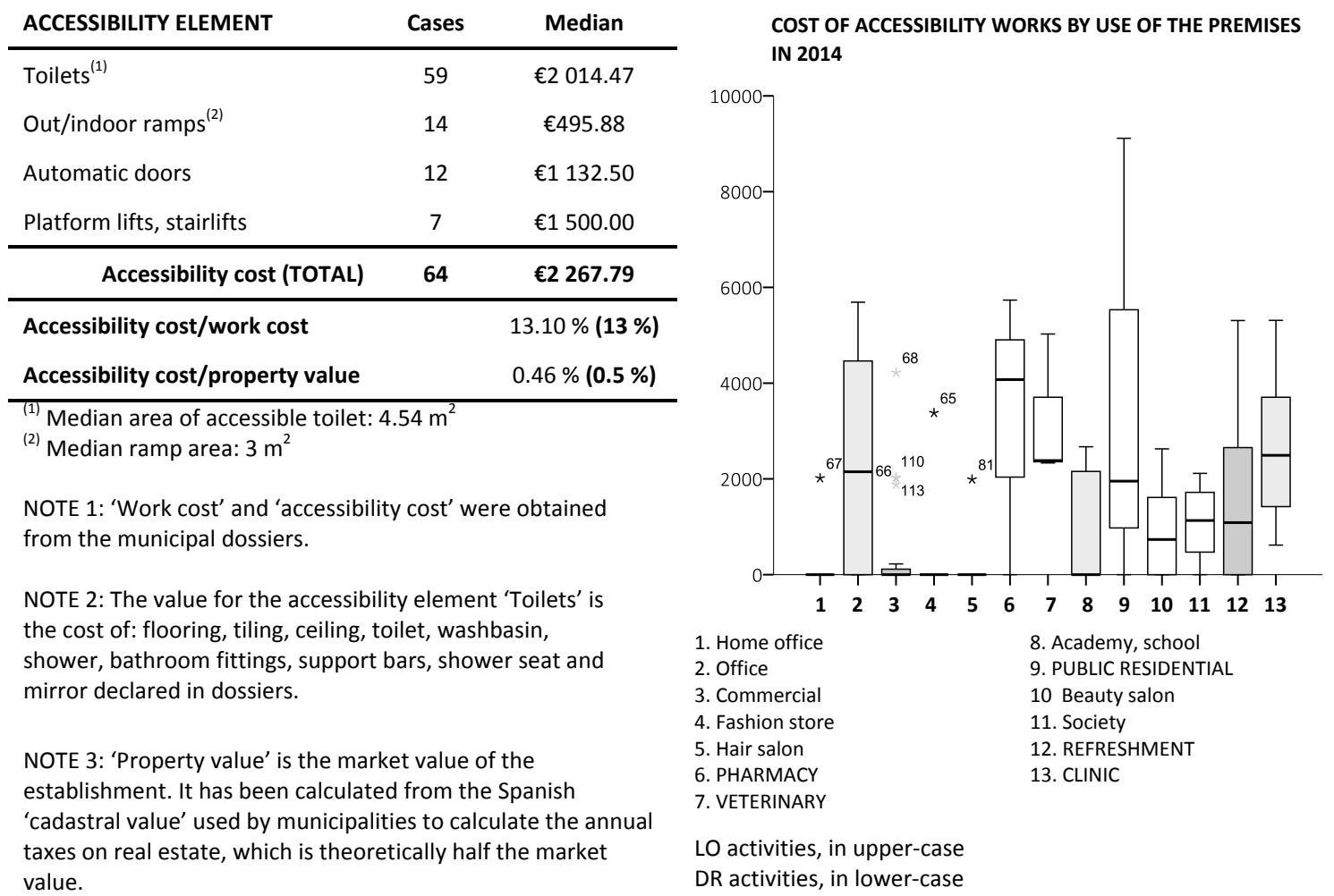

The amounts declared in dossiers, although lower than those established by other construction cost data bases (EME DOS, 2014; CYPE, 2014; COAATG, 2014), have been shown to be homogeneous among different dossiers. The lower the construction costs, the lower are the municipal taxes, and it could be the explanation.

The median cost of accessibility improvements, based on the activities that undertook such works (64 establishments), was around $€ 2250$, essentially intended to improve 'entrances' and 'toilets open to the public'. However, other measures such as signs, information, accessible furniture, etc. have never been budgeted (the latter do not involve very high costs compared to those budgeted, as indicated in Table 1).

Table 8 shows the assessed cost variables, related to the change (NAg_change) and the accessibility level (Nag_pub) by activity, comparing with the obtained results: ' $13 \%$ work cost' and ' $0.5 \%$ property value'.

Table 8. Economic capacity of the owner of the business by activity, ordered by lower value of Nag_pub

\begin{tabular}{|c|c|c|c|c|c|c|c|c|}
\hline & activity & $\begin{array}{l}\text { work cost } \\
\text { (median) }\end{array}$ & $\begin{array}{l}\text { property } \\
\text { value } \\
\text { (median) }\end{array}$ & $\begin{array}{c}\text { accessibility } \\
\text { cost } \\
\text { (median) }\end{array}$ & $\begin{array}{c}\text { NAg_change } \\
\text { (median) }\end{array}$ & $\begin{array}{l}\text { NAg_pub } \\
\text { (median) }\end{array}$ & $\begin{array}{c}13 \% \\
\text { work cost } \\
\text { (A) }\end{array}$ & $\begin{array}{c}0.5 \% \\
\text { property value } \\
\text { (B) }\end{array}$ \\
\hline DR & $\begin{array}{l}\text { hair salon } \\
\text { ( } 7 \text { cases) }\end{array}$ & $\begin{array}{l}€ 5268 \\
66 € / \mathrm{m}^{2}\end{array}$ & $\begin{array}{l}€ 258164 € \\
3229 € / \mathrm{m}^{2}\end{array}$ & $€ 0 €$ & 0 & 0.25 & $€ 685$ & $€ 1291$ \\
\hline DR & $\begin{array}{l}\text { beauty salon } \\
\text { ( } 6 \text { cases) }\end{array}$ & $\begin{array}{l}€ 9022 \\
60 € / \mathrm{m}^{2}\end{array}$ & $\begin{array}{l}€ 371274 € \\
2318 € / \mathrm{m}^{2}\end{array}$ & $€ 734,5$ & 0,5 & 1 & $€ 1173$ & $€ 1856$ \\
\hline DR & $\begin{array}{l}\text { home office } \\
\text { (5 cases) }\end{array}$ & $\begin{array}{c}€ 2015 \\
96 € / \mathrm{m}^{2}\end{array}$ & $\begin{array}{c}€ 127897 \\
3112 € / \mathrm{m}^{2}\end{array}$ & $€ 0$ & 1 & 1.2 & $€ 262$ & $€ 639$ \\
\hline DR & $\begin{array}{l}\text { academy } \\
\text { ( } 9 \text { cases) }\end{array}$ & $\begin{array}{c}€ 5120 \\
32 € / \mathrm{m}^{2}\end{array}$ & $\begin{array}{c}€ 357812 \\
2752 € / \mathrm{m}^{2}\end{array}$ & $€ 0$ & 1 & 1.33 & $€ 666$ & $€ 1789$ \\
\hline DR & $\begin{array}{l}\text { society } \\
\text { (4 cases) }\end{array}$ & $\begin{array}{c}€ 3920 \\
30 € / \mathrm{m}^{2}\end{array}$ & $\begin{array}{c}€ 499015 \\
2721.5 € / \mathrm{m}^{2}\end{array}$ & $€ 1130.5$ & 3.5 & 1.75 & $€ 510$ & $€ 2495$ \\
\hline DR & $\begin{array}{l}\text { commercial } \\
\text { ( } 23 \text { cases) }\end{array}$ & $\begin{array}{c}€ 4304 \\
65 € / \mathrm{m}^{2}\end{array}$ & $\begin{array}{c}€ 210491 \\
2980 € / \mathrm{m}^{2}\end{array}$ & $€ 0$ & 0 & 2 & $€ 560$ & $€ 1052$ \\
\hline DR & $\begin{array}{l}\text { fashion store } \\
\text { ( } 5 \text { cases) }\end{array}$ & $\begin{array}{l}€ 17425 \\
83 € / \mathrm{m}^{2}\end{array}$ & $\begin{array}{c}€ 595694 \\
3685 € / \mathrm{m}^{2}\end{array}$ & $€ 0$ & 3 & 2 & $€ 2265$ & $€ 2978$ \\
\hline LO & $\begin{array}{l}\text { REFRESHMENT } \\
\text { (31 cases) }\end{array}$ & $\begin{array}{c}€ 16879 \\
132 € / \mathrm{m}^{2}\end{array}$ & $\begin{array}{c}€ 548800 \\
3707 € / \mathrm{m}^{2}\end{array}$ & $€ 1087$ & 4 & 2 & $€ 2194$ & $€ 2744$ \\
\hline DR & $\begin{array}{l}\text { office } \\
\text { ( } 9 \text { cases) }\end{array}$ & $\begin{array}{c}€ 25698 \\
105 € / \mathrm{m}^{2}\end{array}$ & $\begin{array}{c}€ 487760 \\
3043 € / \mathrm{m}^{2}\end{array}$ & $€ 2150$ & 3 & 2 & $€ 3341$ & $€ 2439$ \\
\hline
\end{tabular}




\begin{tabular}{|c|c|c|c|c|c|c|c|c|}
\hline & activity & $\begin{array}{l}\text { work cost } \\
\text { (median) }\end{array}$ & $\begin{array}{l}\text { property } \\
\text { value } \\
\text { (median) }\end{array}$ & $\begin{array}{c}\text { accessibility } \\
\text { cost } \\
\text { (median) }\end{array}$ & $\begin{array}{c}\text { NAg_change } \\
\text { (median) }\end{array}$ & $\begin{array}{l}\text { NAg_pub } \\
\text { (median) }\end{array}$ & $\begin{array}{c}13 \% \\
\text { work cost } \\
\text { (A) } \\
\end{array}$ & $\begin{array}{c}0.5 \% \\
\text { property value } \\
\text { (B) }\end{array}$ \\
\hline LO & $\begin{array}{l}\text { VETERINARY } \\
\text { ( } 3 \text { cases) }\end{array}$ & $\begin{array}{c}€ 53556 \\
402 € / \mathrm{m}^{2}\end{array}$ & $\begin{array}{c}€ 513959 \\
2666 € / \mathrm{m}^{2}\end{array}$ & $€ 2383$ & 7 & 2.33 & $€ 6962$ & $€ 2570$ \\
\hline LO & $\begin{array}{l}\text { P.RESIDENTIAL } \\
\text { ( } 3 \text { cases) }\end{array}$ & $\begin{array}{c}€ 9269 \\
56 € / \mathrm{m}^{2}\end{array}$ & $\begin{array}{c}€ 288036 \\
2400 € / \mathrm{m}^{2}\end{array}$ & $€ 1952$ & 3 & 2.5 & $€ 1205$ & $€ 1440$ \\
\hline LO & $\begin{array}{l}\text { CLINIC } \\
\text { (12 cases) }\end{array}$ & $\begin{array}{c}€ 26730.5 \\
196.5 € / \mathrm{m}^{2}\end{array}$ & $\begin{array}{c}€ 602154 \\
3108.5 € / \mathrm{m}^{2}\end{array}$ & $€ 2493.5$ & 5.5 & 2.33 & $€ 3475$ & $€ 3011$ \\
\hline LO & $\begin{array}{l}\text { PHARMACY } \\
\text { (3 cases) }\end{array}$ & $\begin{array}{c}€ 28068 \\
170 € / \mathrm{m}^{2}\end{array}$ & $\begin{array}{c}€ 524673 \\
3094 € / \mathrm{m}^{2}\end{array}$ & $€ 4074$ & 9 & 3 & $€ 3648$ & $€ 2623$ \\
\hline
\end{tabular}

For the analysed sample (Table 7), criteria for measuring the economic feasibility of undertaking the reasonable adjustment required, and therefore the owner's obligation to do so, could be based on the following considerations.

- (A) The accessibility improvements should not exceed $13 \%$ of the initial remodel investment, the median accessibility investment relative to the total cost found for the sample analysed.

- (B) The accessibility improvements should not exceed $0.5 \%$ of the property value, the median accessibility investment relative to the property value found for the sample analysed.

Although criterion A is closer to the accessibility cost (as shown in Table 8), criterion B could be used in the case that only accessibility works are carried out.

The foregoing is similar to the approach proposed in certain regional and international regulations related to Continental European policies, in which accessibility obligations are defined in proportion to total remodel cost or property value (see Section 2, Switzerland legislation).

\subsection{Factors affecting overall accessibility (Nag_pub Index)}

'Decision-tree multivariate classification' was found to be the method best suited to the variables assessed. Decision trees group cases and predict dependent variable values on the grounds of explanatory/predictive variable values.

For applying this test, the continuous dependent variable (NAg_pub) was categorized into 3 ordinal values by range: inaccessible, intermediate and accessible. Figure 5 shows the criteria used for these categories. Scope and proportionality variables (Figure 2) were assessed as explanatory values.
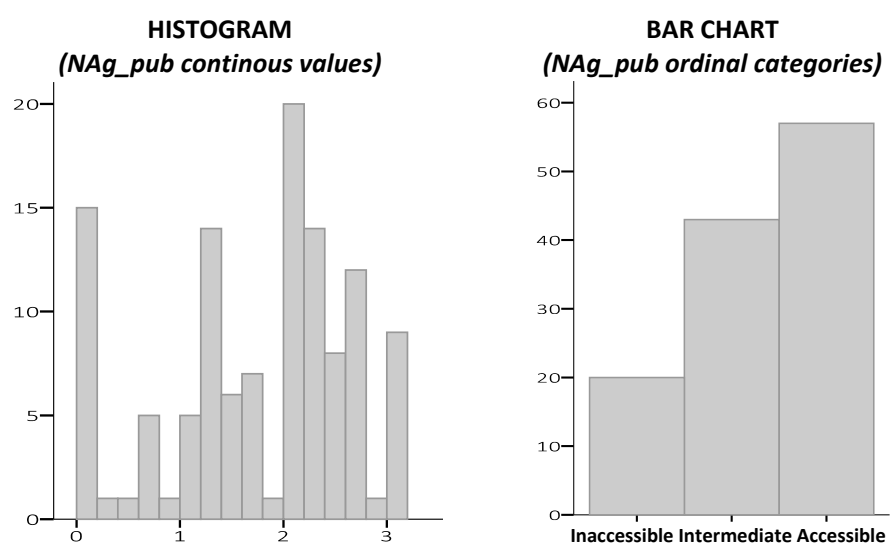

RANGES

NAg_pub ranges

$\begin{array}{lc}\text { Inaccessible values } & 0-0.99 \\ \text { Intermediate values } & 1-1.99 \\ \text { Accessible values } & 2-3\end{array}$

Figure 5: NAg_pub Index: overall accessibility index in areas open to the public

The decision tree findings depicted in Figure 6 and summarised in Table 9 afforded an optimal explanation of the results for the overall accessibility index in areas open to the public (Nag_pub Index) with a $70 \%$ goodness of fit for the accessibility category. 


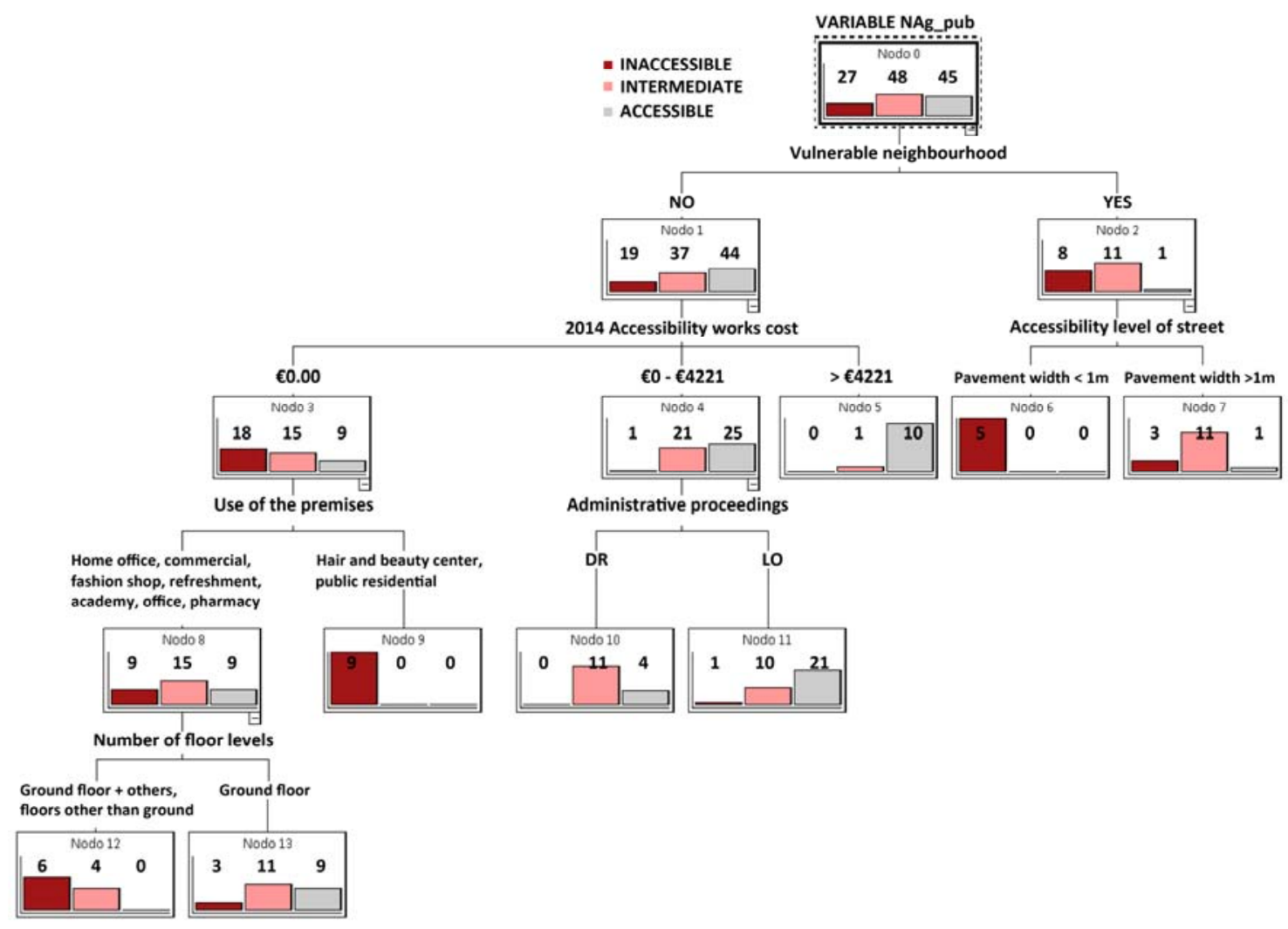

Figure 6. Decision tree for accessibility improvements in areas open to the public. Nonparametric tests (SPSS Statistics software)

Table 9: Observed and predicted classification of variable 'NAg_pub Index', by accessibility category

\begin{tabular}{lcccc}
\hline \multirow{2}{*}{ Observed } & \multicolumn{4}{c}{ Predicted } \\
\cline { 2 - 5 } & Inaccessible & Intermediate & Accessible & $\begin{array}{c}\text { \% correctly } \\
\text { predicted }\end{array}$ \\
\hline Inaccessible & 20 & 6 & 1 & $74.1 \%$ \\
\hline Intermediate & 4 & 33 & 11 & $68.8 \%$ \\
\hline Accessible & 0 & 14 & 31 & $68.8 \%$ \\
\hline$\%$ of cases classified overall & $20 \%$ & $44.2 \%$ & $35.8 \%$ & $\mathbf{7 0 \%}$ \\
\hline$\%$ total & $22 \%$ & $40 \%$ & $38 \%$ & $100 \%$ \\
\hline
\end{tabular}

CHAID tree-growing method

The resultant 'explanatory variables' affecting the possibility of adapting areas open to the public in small establishments included the following.

- In vulnerable neighbourhoods ${ }^{2}$, the most significant factor was pavement (walkway) width, intermediate accessibility categories were obtained for premises located alongside pavements over $1.5 \mathrm{~m}$ wide, whilst in all other cases the establishments were inaccessible.

- In non-vulnerable neighbourhoods, the most significant factor was the cost of accessibility works.

- High accessibility was obtainable when the budget was in excess of €4221.

- High accessibility categories could be attained at intermediate cost where the works were subject to the most demanding municipal administrative proceedings (LO). Where less demanding proceedings were involved (DR), intermediate accessibility was more likely.

\footnotetext{
${ }^{2}$ Vulnerable neighbourhoods: The Observatory of Urban Vulnerability is a long-term project of the Ministry of Public Works, which is intended to accommodate various studies related to urban vulnerability in Spain. This Observatory contains, among other tools, the 'Atlas of Urban Vulnerability' with information at the census section level of all Spanish municipalities, as well as the 'Catalogue of Vulnerable Neighbourhoods'. https://www.fomento.gob.es/areas-de-actividad/arquitectura-vivienda-y-suelo/urbanismo-y-politica-desuelo/observatorio-de-la-vulnerabilidad-urbana/analisis-urbanistico-de-barrios-vulnerables
} 
- When accessibility works were not undertaken, the existing accessibility conditions were the result of the premises, of a single storey, in which the entrance was not stepped or stepped by less than $20 \mathrm{~cm}$. In other cases, the establishments were accessible prior to the works.

In light of the foregoing, the following urban planning criteria are proposed for adoption by the authorities when establishing reasonable adjustment requirements:

- When the public thoroughfare is not accessible (as defined in Section 3.3.1), establishments should not be required to undertake adaptations until the measures needed to remedy that situation are implemented by municipalities. Consequently, adaptation in urban areas shall be efficiently planned.

- When an establishment is on a storey of a building connected to the public thoroughfare via an inaccessible itinerary (as defined in section 3.3.1) and the owner of the premises is not the sole owner of the building, until such time as the itinerary can be adapted:

- no adaptations should be required in the premises;

- change of use or extension to premises should not be allowed, except where the new use is not intended for people with disabilities; if the public thoroughfare or indoor itinerary in the building is adapted to some manner of specific disability, the premises should be required to adapt its facilities to that disability.

Consequently, incentives or other types of government aid should be granted for the adaptation of communal areas (similarly to subsidies in the Spanish residential scope).

\subsection{Municipal policies. Overall accessibility by city (Nag_pub and Nag_change Indexes)}

The pre- and post-adaptation solutions for the variable NAg_pub Index were overlaid on the maps of the cities studied (Figure 7), highlighting the establishments lying in the two extreme categories (NAg_pub Index = 'O' meaning no accessible elements and NAg_pub Index='3' accessibility-compliant).
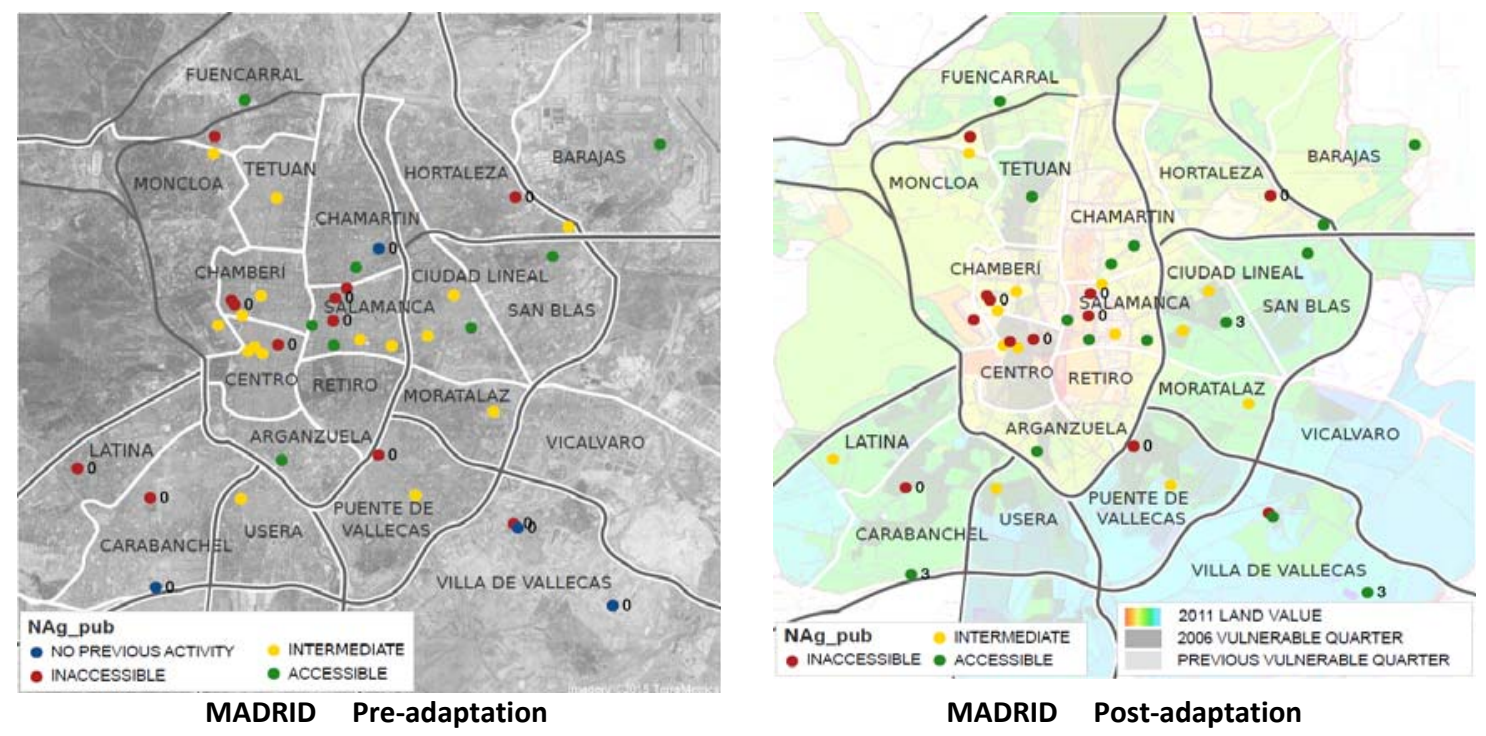


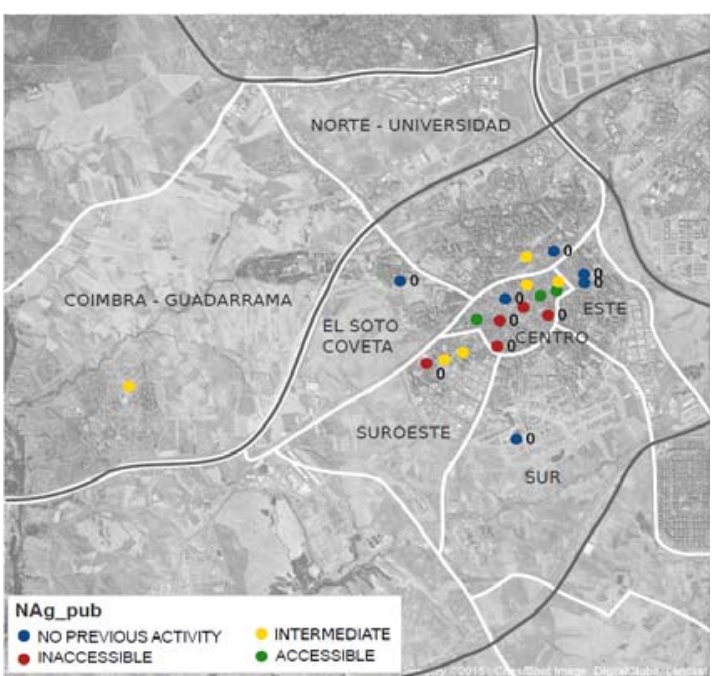

MOSTOLES Pre-adaptation

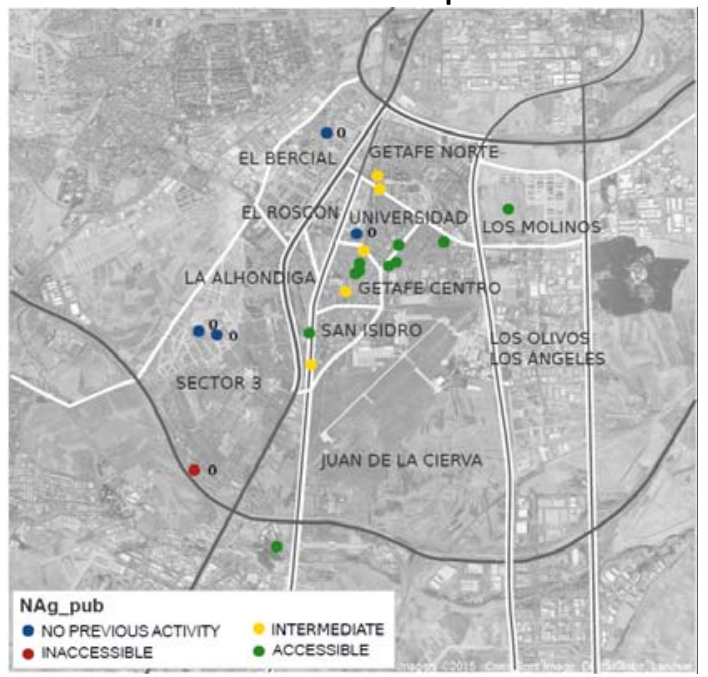

GETAFE Pre-adaptation

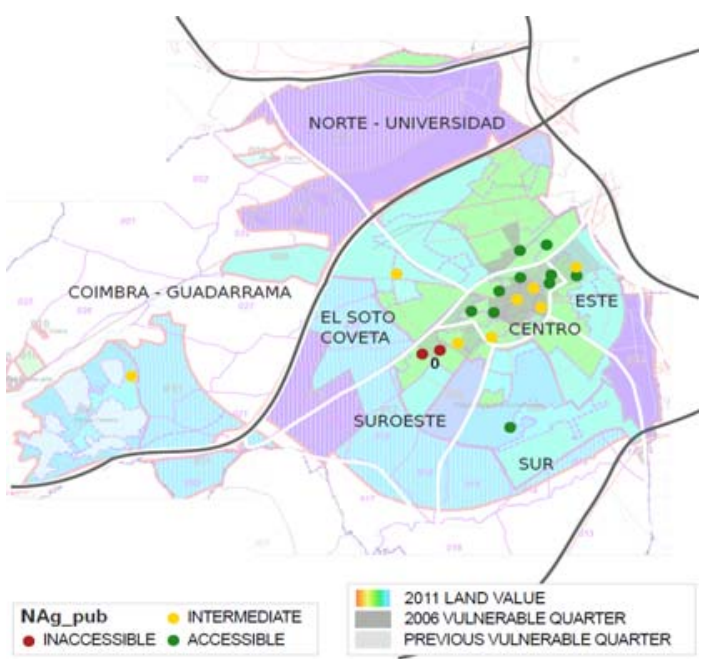

MOSTOLES Post-adaptation

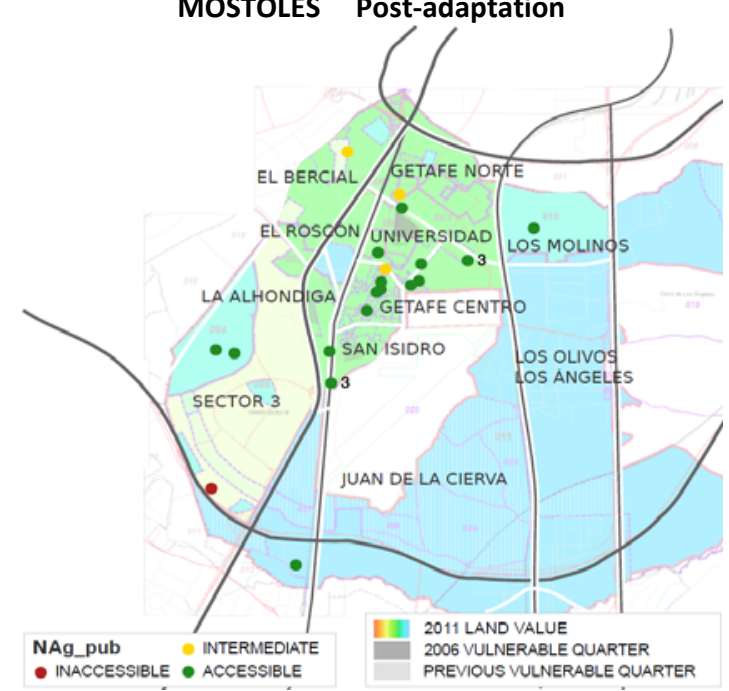

GETAFE Post-adaptation

Figure 7. Location of establishments in highest and lowest accessibility categories (variable NAg_pub), pre- and post-adaptation; example for Madrid, Mostoles and Getafe.

Map sources:

(1) Vulnerable quarters: https://www.fomento.gob.es/areas-de-actividad/arquitectura-vivienda-y-suelo/urbanismoy-politica-de-suelo/observatorio-de-la-vulnerabilidad-urbana/analisis-urbanistico-de-barrios-vulnerables

(2) Land value: http://www.catastro.meh.es/esp/wms.asp

Lower accessibility scores were observed in quarters with urban shortcomings, older buildings and symptoms of vulnerability. Activities investing scantly in remodelling and therefore in accessibility works were located in these quarters, with previously configured premises. Conversely, premises initially consisting in raw spaces ('no previous activity' establishment, see Figure 4) invested most in remodelling (identified in blue in Figure 7) and exhibited the best accessibility results. Again, vulnerable quarters represented by historic centres, spontaneous growth areas and workers' neighbourhoods, are shown as the least adapted.

Whilst the greatest improvement was recorded for Móstoles (NAg_change Index=6, Table 6), accessibility (Nag_pub index) scores were higher in Getafe, even in establishments located in the city centre. The initial accessibility scores were higher in Getafe than in any of the other cities, perhaps because of general accessibility improvements had been progressively introduced in urban planning.

Accessibility improvements were lowest in Madrid (mean NAg_change Index=2, table 10). These results might be associated with a municipal edict (Instrucción 4/2011, artículo 3) whereby the non-viability of adaptations to meet accessibility requirements can be based on generic and non-quantified criteria. These results might also be attributed to the outsourcing of administrative proceedings to private licensing agencies, precluding the ability to address queries to the public authorities directly. 
Table 10. Number of dossiers by accessibility category (NAg_pub Index) and city, pre- and post-adaptation; variable 'NAg_change Index ${ }^{\text {(1) }}$ by city

\begin{tabular}{lcclcc}
\hline NAg_pub Index & Pre-adaptation & Post-adaptation & NAg_pub Index & Pre-acc. & Post-acc. \\
\hline MADRID & (NAg_change Index=2) & & GETAFE & (NAg_change Index=4) & \\
\hline Accessible & 8 & 10 & Accessible & 10 & 12 \\
intermediate & 16 & 17 & intermediate & 5 & 7 \\
Inaccessible & 16 & 13 & Inaccessible & 5 & 1 \\
\hline MOSTOLES & $\left(N A g \_c h a n g e ~ I n d e x=6\right)$ & VALDEMORO & $($ NAg_change Index=4) \\
\hline Accessible & 3 & 8 & Accessible & 4 & 6 \\
intermediate & 6 & 9 & intermediate & 4 & 7 \\
Inaccessible & 11 & 3 & Inaccessible & 12 & 7 \\
\hline LAS ROZAS & $($ NAg_change Index=4) & TOTAL & & \\
\hline Accessible & 9 & 9 & Accessible & $\mathbf{2 6 \%}$ & $\mathbf{3 8 \%}$ \\
intermediate & 5 & 8 & intermediate & $\mathbf{3 0 \%}$ & $\mathbf{4 0 \%}$ \\
Inaccessible & 6 & 3 & Inaccessible & $\mathbf{4 4 \%}$ & $\mathbf{2 2} \%$ \\
\hline
\end{tabular}

(1) NAg_change Index: average value of the variable 'NAg_change' (Section 3.3.2), calculated by city. Nag_change Index measures the overall difference between the initial and final accessibility results of establishments, by city.

\section{Conclusions. The need for a strategy to improve access}

Existing cities, conceived with different functional criteria than today, have a large number of barriers to access, which requires extended times to adapt. The United Nations Convention of 2006 and, particularly in Spain, the General Act on the Rights of Persons with Disabilities constitute a challenge to realize this objective.

Differently from legal actions by private individuals in employment and housing cases, a greater coordination and a planned policy are urgently demanded to make this framework effective, prioritizing basic needs based on a comprehensive and transparent assessment of the existing environment. This study shows a methodological example for a case study in Madrid and its metropolitan area.

In the sphere of establishments, this study discusses a system of accessibility variables to define the concept of 'reasonable adjustment' in the context of the challenge posed by the 2017 Spanish deadline (Real Decreto Legislativo 1/2013). As shown, interpretation and application of the regulations are more difficult in small establishments, which constitute most of the built environment with accessibility shortcomings. In order to define this concept according to the 'continental European model', a regulatory framework could be developed based on variables as analyzed here.

Following this study, technical solutions and flexibility considerations have already been assumed in the Spanish Building Code (DA DB SUA/2, 2015). However, specific variables related to cost, vulnerability conditions, urban planning, etc., should be taken into account in legislation, similarly to Spanish residential, French strategies and Swiss legislative economical criteria, described in Section 2.

The defined variables of this study address:

- Flexibility and applicability of technical accessibility conditions (Section 3.3 and 3.3.1). The flexibility provisions set out in technical codes are only needed for wheelchair-accessibility adaptations, namely to manoeuvre and to move vertically, which entail greater technical difficulty and cost than other accessibility requirements. The absence of solutions for adapting premises to these users does not, however, justify the non-compliance (regrettably observed in many of the dossiers analysed) with requirements relating to people with other disabilities, which can be readily implemented.

- Viability of improving entrances (NA_D_AEdif) and toilets open to the public (NA_As_pub), the most characteristic elements of these spaces difficult to adapt. On the whole, compliance with accessibility requirements was found to lack rigour, for efforts that could have delivered optimal results failed to do so. Non-compliance was often attributable more to insufficient understanding of the legislation than any intended breach of its provisions. In other situations, the study detected indifference to adapt a previously configured element inaccessible, revealing the importance of 
accessibility training, even today. Promoting an awareness campaign is necessary to successfully address accessible environments.

- Economic viability of improvements in keeping with the type of activity (cost variables). For this case study, the economic capacity of the business owner, as justified in the administrative dossiers, has confirmed that accessibility improvements should not exceed either $13 \%$ of the initial remodel investment or $0.5 \%$ of the property value.

- Improvements in overall accessibility (Nag_pub Index applied in 'decision tree' tests). For this case study, communal areas in buildings and the public thoroughfare, spaces that can not be adapted by the owner of the property, have been revealed as critical when accessibility works are carried out. Therefore, the main efforts should focus on adapting these points.

Validating earlier articles, high levels of non-compliance are concentrated in vulnerable neighbourhoods, including historic city centres. Besides, in certain types of activities owners invest less in works. In these inaccessible environments, where the more aged population with fewer resources is located, Public Authorities should prioritise incentives and renovations in urban planning, in order to promote and make easier adaptations in establishments. Decision tree tests (as developed in Section 4.3) and cost analysis by type of activity (as developed in Section 4.2) could guide these decisions.

The difference between the most (LO) and the least strict (DR) administrative permit was significant in all accessibility results. In Spain, the lack of control over DR permits and their consequences on poor accessibility scores are severely criticised by municipal control authorities, who demand a regulatory amendment (Rodriguez, 2014).

Additionally, municipal policies were evaluated, demonstrating that their action also conditions accessibility scores. The most satisfactory results were observed in cities exhibiting a greater concern for remedying accessibility shortcomings in urban planning. Lower scores were recorded for cities where non-viability allegations can be based on general, non-quantified criteria, for designers seize on such guidelines to readily justify non-compliance with no incentive to seek alternatives. The existence of intermediaries between the licence applicant and the municipal surveyor has also led to the deployment of less effort to conform to regulatory standards.

The foregoing may serve as a reference for public policy on accessibility conditions in this specific scope in order to design a strategy based on progressive priorities of accessibility (short and long-term) in the existing built environment, which would redound to better living conditions for a particularly vulnerable sector of society and their more effective exercise of an inalienable right.

\section{Acknowledgments}

\section{References}

Alegre, L.; Casado, N.; Vergés, J. (2005). Documentos 47/2005: Análisis comparado de las normas estatales y autonómicas de accesibilidad. Real Patronato sobre Discapacidad, Madrid.

http://sid.usal.es/libros/discapacidad/12663/8-1/analisis-comparado-de-las-normas-autonomicas-yestatales-de-accesibilidad.aspx (Accessed February 2019)

Berrigan, D.; Troiano, R. P. (2002). The association between urban form and physical activity in U.S. adults. American Journal of Preventive Medicine, 23(2), 74-79.

https://doi.org/10.1016/S0749-3797(02)00476-2

Bosch, J. (2013). Ciudad y envejecimiento: bases para un nuevo urbanismo. V Premio Nacional de Urbanismo Ricardo Santos Diez. Práctica urbanística: Revista mensual de urbanismo, 120, 36-51

Bromley, R. D., Matthews, D. L., Thomas, C. J. (2007). City centre accessibility for wheelchair users: The consumer perspective and the planning implications. Cities, 24(3), 229-241.

https://doi.org/10.1016/j.cities.2007.01.009 
Burkhauser, R. V., Butler, J. S., Kim, Y. W. (1995). The importance of employer accommodation on the job duration of workers with disabilities: A hazard model approach. Labour Economics, 2(2), 109-130.

https://doi.org/10.1016/0927-5371(95)80049-4

Cabra de Luna, M. A. (2004). Discapacidad y aspectos sociales: la igualdad de oportunidades, la no discriminación y la accesibilidad universal como ejes de una nueva política a favor de las personas con discapacidad y sus familias. Algunas consideraciones en materia de protección social, Revista del Ministerio de Trabajo e Inmigración, 50, 21-46.

http://sid.usal.es/articulos/discapacidad/6696/8-2-6/discapacidad-y-aspectos-sociales-la-igualdad-deoportunidades-la-no-discriminacion-y-la-accesibilidad-universal-como-ejes-de-una-nueva-politica-afavo.aspx (Accessed February 2019)

Campion,C. L. (2013). Réussir 2015. Accessibilité des personnes handicapées au logement, aux établissements recevant du public, aux transports, à la voirie et aux espaces publics https://www.ecologique-solidaire.gouv.fr/sites/default/files/Campion reussir\%202015.pdf (Accessed February 2019)

COAATG (2014). Base de precios centro (2014). Colegio oficial de arquitectos técnicos Guadalajara. http://preciocentro.com/base-de-precios

CYPE (2014). Generador de precios de construcción, España (2014). CYPE Ingenieros SA. http://www.generadordeprecios.info.

De Campos Velho Martel, L. (2011). Reasonable Accommodation: The New Concept from an Inclusive Constitutional Perspective. Sur - International Journal on Human Rights, 14, 85-111.

https://papers.ssrn.com/sol3/papers.cfm?abstract id=2000291 (Accessed February 2019)

Del Moral, C.; et. Al. (2015). La accesibilidad en la vivienda: sistema integrado de diagnosis e intervención. 5o Congreso de Patología y Rehabilitación de Edificios. PATORREB 2015, Oporto, 2015.

http://www.aopandalucia.es/innovacion/principal.asp?alias=Vivienda accesible sostenible (Accessed February 2019)

Disability Right Commission (2005). Making access to goods and services easier for disabled customers. A practical guide for small service providers.

www.equalitylink.co.uk/downloads/Access to Goods and Services.pdf (Accessed February 2019)

EME DOS (2014). Índices EMEDOS de costes de la construcción. Trifolio ediciones S.L. 4o trimestre (154).

Equality and Human Rights Commission (2011). Equality Act 2010 Code of Practice. Services, public functions and associations. Statutory Code of Practice.

https://www.equalityhumanrights.com/en/publication-download/services-public-functions-andassociations-statutory-code-practice (Accessed February 2019)

European Union (2002). Europe's response to World Ageing. http://eur-lex.europa.eu/legalcontent/EN/TXT/?uri=LEGISSUM:c11910 (Accessed February 2019)

Froehlich-Grobe, K., Regan, G., Reese-Smith, J. Y., Heinrich, K. M., Lee, R. E. (2008). Physical access in urban public housing facilities. Disability and Health Journal, 1(1), 25-29.

https://doi.org/10.1016/j.dhjo.2007.11.003

Fundación ONCE. (2013). Observatorio de la accesibilidad en los municipios de España 2011. Fundación Once. http://riberdis.cedd.net/handle/11181/3867 (Accessed February 2019)

Fundación ONCE (2015). Observatorio de la accesibilidad universal en la vivienda en España 2013.

Fundación Once. http://riberdis.cedd.net/handle/11181/4630 (Accessed February 2019)

Jaraba, M, (2012). La estrategia europea de discapacidad 2010-2020: nuevas herramientas contra la discriminación. In: 2003-2012, 10 años de legislación sobre no discriminación de personas con 
discapacidad en España. CERMI, 154-177

http://riberdis.cedd.net/handle/11181/4274 (Accessed February 2019)

Kirchner, C. E., Gerber, E. G., Smith, B. C. (2008). Designed to Deter. American Journal of Preventive Medicine, 34(4), 349-352. https://doi.org/10.1016/j.amepre.2008.01.005

Lansley, P., Flanagan, S., Goodacre, K., Turner-Smith, A., Cowan, D. (2005). Assessing the adaptability of the existing homes of older people. Building and Environment, 40(7), 949-963.

https://doi.org/10.1016/j.buildenv.2004.09.011

Lewis, C., McQuade, J., \& Thomas, C. (2005). Measuring physical access barriers to services: 'Snapshot' research in 4 town/city centres in Britain. International Congress Series, 1282, 1034-1037.

https://doi.org/10.1016/j.ics.2005.05.184

Madrid (2011). La ciudad de Madrid en cifras 2011. Área de Gobierno de Hacienda y Administración Pública. Dirección general de estadística.

https://www.madrid.es/UnidadesDescentralizadas/UDCEstadistica/Nuevaweb/Publicaciones/Madrid\%20 en\%20Cifras/Madrid\%20en\%20Cifras\%202011.pdf (Accessed February 2019)

Madrid (2014). Estadísticas de licencias urbanísticas. Enero 2015. Área de Gobierno de Urbanismo y Vivienda. Departamento de información geográfica.

Manley, S. (1996). Walls of exclusion: the role of local authorities in creating barrier-free streets. Landscape and Urban Planning, 35(2-3), 137-152. https://doi.org/10.1016/0169-2046(96)00310-6

Meager, N., Dewson, S., Evans, C., Harper, H. (2002). Costs and Benefits to Service Providers of Making Reasonable Adjustments under Part III of the Disability Discrimination Act. Research Report DWPRR 169. Department for Work and Pensions. http://www.employment-studies.co.uk/resource/costs-and-benefitsservice-providers-making-reasonable-adjustments-under-part-iii (Accessed May 2017)

Michael, Y.L.; Green, M.K.; Farquhar, S.A. (2006) Neighborhood design and active aging, Health \& Place, 12(4): pp. 734-740. https://doi.org/10.1016/j.healthplace.2005.08.002

Observatorio Estatal de la Discapacidad. (2012). Las personas con discapacidad en España. Informe Olivenza 2010. http://riberdis.cedd.net/handle/11181/3422 (Accessed May 2017)

Oliva, J. (2015) Marco legislativo y reglamentario: Cataluña. Un ejemplo a nivel autonómico. In: Libro de ponencias del Seminario 5: accesibilidad y ajustes razonables en edificación existente (II). Cursos avanzados Eduardo Torroja. Publicaciones del IETcc-CSIC, 29-40. ISBN: 978-84-7292-389-8

Pérez Bueno, L. C. (2012). La configuración jurídica de los ajustes razonables. In: 2003-2012, 10 años de legislación sobre no discriminación de personas con discapacidad en España. CERMI, 178-209. http://riberdis.cedd.net/handle/11181/4274 (Accessed February 2019)

Prideaux, S. (2006) Good Practice for Providing Reasonable Access to the Physical Built Environment for Disabled People. Centre for Disability Studies, University of Leeds. http://disability-

studies.leeds.ac.uk/research/good-practice-for-providing-reasonable-access-to-the-physical-builtenvironment-for-disabled-people/ (Accessed February 2019)

Prost-Coletta, M. (2015) Marco legislativo y reglamentario: Francia. Un ejemplo a nivel internacional. In: Libro de ponencias del Seminario 5: accesibilidad y ajustes razonables en edificación existente (II). Cursos avanzados Eduardo Torroja. Publicaciones del IETcc-CSIC, 21-28. ISBN: 978-84-7292-389-8

Rodríguez, R. (2014). La adaptación de establecimientos a la accesibilidad. Una visión municipal Libro de ponencias del Seminario 5: accesibilidad y ajustes razonables en edificación existente. Cursos avanzados Eduardo Torroja. Publicaciones del IETcc-CSIC, 145-152. ISBN: 978-84-7292-427-7 
Roulstone, A., Prideaux, S. (2009). Constructing reasonableness: Environmental access policy for disabled wheelchair users in four European Union countries. ALTER - European Journal of Disability Research / Revue Européenne de Recherche Sur Le Handicap, 3(4), 360-377.

https://doi.org/10.1016/j.alter.2009.03.004

Serrano, A., Barrios, Á., Molina, M. (2018). Sustainable building renovation for an ageing population: Decision support system through an integral assessment method of architectural interventions.

Sustainable Cities and Society, 39, 144-154. https://doi.org/10.1016/i.scs.2018.01.050

Sixsmith, A.; Sixsmith, J. (2008). Ageing in Place in the United Kingdom. Ageing International, 32 (3), 219 235, 2008. https://doi.org/10.1007/s12126-008-9019-y

Solovieva, T. I., Walls, R. T., Hendricks, D. J., \& Dowler, D. L. (2009). Cost of workplace accommodations for individuals with disabilities: with or without personal assistance services. Disability and Health Journal, 2(4), 196-205. https://doi.org/10.1016/j.dhjo.2009.04.002

Solovieva, T. I., Dowler, D. L., \& Walls, R. T. (2011). Employer benefits from making workplace accommodations. Disability and Health Journal, 4(1), 39-45. https://doi.org/10.1016/j.dhjo.2010.03.001

Tecnalia (2011). Diagnóstico de las necesidades de intervención en la renovación del parque edificado del País Vasco. Departamento de vivienda, obras públicas y urbanismo, Gobierno Vasco.

http://www.etxebide.euskadi.eus/contenidos/nota prensa/npetxe120307 inventario parque/es npetxe Ladjuntos/informe.pdf (Accessed February 2019)

United Nations (2005). The Concept of Reasonable Accommodation in Selected National Disability Legislation. Background conference document prepared by the Department of Economic and Social Affairs. http://www.un.org/esa/socdev/enable/rights/ahc7bkgrndra.htm (Accessed February 2019)

Vega, L.; Posada, J. L. (2012). Consideraciones de la Administración sobre la aplicación de la reglamentación a edificios existentes. Informes de la Construcción, 64 (Extra), 135-140. https://doi.org/10.3989/ic.11.080

Waddington, L. (2008). When it is Reasonable for Europeans to Be Confused: Understanding When a Disability Accommodation is 'Reasonable' from a Comparative Perspective (SSRN Scholarly Paper No. ID 1128295). Rochester, NY: Social Science Research Network. https://papers.ssrn.com/abstract=1128295 (Accessed February 2019)

World Health Organization (2015). World report on ageing and health. http://apps.who.int/iris/bitstream/10665/186463/1/9789240694811 eng.pdf. (Accessed February 2019)

World Health Organization (2007). Global Age-friendly Cities: A Guide. World Health Organization. https://www.who.int/ageing/publications/Global age friendly cities Guide English.pdf (Accessed February 2019)

Women and Equalities Committee (2017). Building for equality: Government must lead the charge. Parliament of United Kingdom. https://www.parliament.uk/business/committees/committees-az/commons-select/women-and-equalities-committee/news-parliament-2015/disability-and-builtenvironment-report-published-16-17/ (Accessed February 2019) 